\title{
A Practical and General Synthesis of Unsymmetrical Terphenyls
}

Jose M. Antelo Miguez, ${ }^{\dagger}$ Luis Angel Adrio, Antonio Sousa-Pedrares, ${ }^{\dagger}$ Jose M. Vila, ${ }^{\dagger}$ King Kuok (Mimi) Hii*

Department of Chemistry, Imperial College London, Exhibition Raod, South Kensington, London SW7 2AZ, United Kingdom.

\section{Table of Content}

General Experimental $\mathrm{S} 2$

Characterisation data for compounds $2 \mathbf{a}-\mathbf{2 m}, \mathbf{3 a}-\mathbf{3 k}, \mathbf{4 a - 4 k}$ and $\mathbf{5 a - 5 j}$. S3-S15

Figure S1 and S2. ORTEP drawing and crystal packing of compound 3k. S16

Table S1. Crystal data and structure refinement for compound 3k. S16-S17

Table S2. Bond lengths $[\AA]$ and angles $\left[^{\circ}\right]$ for compound $3 \mathbf{k}$ $\mathrm{S} 18-\mathrm{S} 21$

Table S3. UV absorption maxima of the terphenyl compounds. S22-S23

Figure S2-S22. ${ }^{1} \mathrm{H}$ and ${ }^{13} \mathrm{C}$ NMR spectra of compounds 3a, 3d, 3e, 3g, 3h, 3k, 4a, 4c-4h, 4j, 4k, $5 e-5 g$. S24-S44

References S45-46

Departamento de Química Inorgánica, Facultad de Química, Universidad de Santiago de Compostela, E-15782, Spain. 
General Experimental. Bromochlorobenzene, arylboronic acid precursors and all other reagents were procured from commercial suppliers and used as received. Palladium acetate was obtained via a precious metal loan from Johnson Matthey plc. Catalytic reactions were generally conducted on a Radley's 12-place reaction carousel. Solid samples for infrared spectroscopy were formulated as $\mathrm{KBr}$ discs, whereas liquid samples were recorded as thin films between $\mathrm{NaCl}$ plates.

Crystal Structure Determination. Three-dimensional X-ray data were collected at 293(2) K in the range $1.55<\theta<23.96^{\circ}$ on a Bruker Smart CCD diffractometer by the $\omega$-scan method using graphite-monochromated Mo $\mathrm{K}_{\alpha}$ radiation $(\lambda=0.7107 \AA)$. The structure of compound 3k was solved by SIR-97 and refined anisotropically (SHELXL-97) by full-matrix blocked least squares on $F^{2}$ with allowance for the thermal anisotropy of all non-hydrogen atoms. Refinement converged at a final $R=0.1175\left(R_{\mathrm{w}}=0.2499\right)$. The structure solution and refinement were carried out using the program package SHELX-97. Crystal data and details on data collection and refinement for compound 3k: $\mathrm{C}_{20} \mathrm{H}_{18} \mathrm{O}, \mathrm{Fw}=274.34$, monoclinic, $P 2_{1} / c, a=7.601(5) \AA, b$ $=7.577(5) \AA, c=26.341(5) \AA, \beta=94.107(5)^{\circ}, V=1513.2(2) \AA^{3}, Z=4$. A total of 9325 reflections were measured, with 2358 having $I>2 \sigma(I)$. The $\theta$ range was $1.55-23.96^{\circ}$ with $h k l$ indices of -8 to $8,-8-8$, and $-29-29$. An absorption correction was applied $\left(\mu=0.072 \mathrm{~mm}^{-1}\right.$, 0.976-0.986 transmission). $R$ values $(I>2 \sigma(I)): \mathrm{R} 1=0.0819$, wR2 $=0.2257 . R$ values (all reflections): $\mathrm{R} 1=0.1175, \mathrm{wR} 2=0.2499$. GOF $=1.130$. The residual electron density was between -0.233 and $0.294 \mathrm{e} / \AA^{3}$.

Crystallographic data (excluding structure factors) for the compound $3 \mathbf{k}$ have also been deposited with the Cambridge Crystallographic Data Centre as supplementary publication no. CCDC-642531. Copies of the data can be obtained free of charge on application to CCDC, 12 Union Road, Cambridge CB2 1EZ, UK [Fax: int. code + 44(1223)336-033; E-mail: deposit@ccdc.cam.ac.uk].

1-(4'-Chlorobiphenyl-4-yl)ethanone, 2a. White solid. mp 104-105 ${ }^{\circ} \mathrm{C}$ (lit. ${ }^{1} 103-104{ }^{\circ} \mathrm{C}$, methanol). $\delta_{\mathrm{H}}\left(\mathrm{CDCl}_{3}, 300 \mathrm{MHz}\right): 2.64(3 \mathrm{H}, \mathrm{s}), 7.44(2 \mathrm{H}, \mathrm{d}, J 8.3 \mathrm{~Hz}), 7.56(2 \mathrm{H}, \mathrm{d}, J 8.3 \mathrm{~Hz}), 7.65$ $(2 \mathrm{H}, \mathrm{d}, J 8.3 \mathrm{~Hz}), 8.03(2 \mathrm{H}, \mathrm{d}, J 8.3 \mathrm{~Hz}) . \delta_{\mathrm{C}}\left(\mathrm{CDCl}_{3}, 75.5 \mathrm{MHz}\right): 26.9,127.3(2 \mathrm{C}), 128.7(2 \mathrm{C}), 129.2$ (2C), 129.4 (2C), 134.7, 136.3, 138.5, 144.6, 197.8. m/z (EI) $230\left(\mathrm{M}^{+}, 23 \%\right), 215$ (50), 187 (2), 152 (44), $76(13), 58$ (100). 
1-(3'-Chlorobiphenyl-4-yl)ethanone, 2b. White solid. mp 54-56 ${ }^{\circ} \mathrm{C}$ (lit. ${ }^{1} 52-53{ }^{\circ} \mathrm{C}$, methanol). $\delta_{\mathrm{H}}$ $\left(\mathrm{CDCl}_{3}, 300 \mathrm{MHz}\right): 2.64(3 \mathrm{H}, \mathrm{s}), 7.36-7.42(2 \mathrm{H}, \mathrm{m}), 7.49-7.51(1 \mathrm{H}, \mathrm{m}), 7.59-7.61(1 \mathrm{H}, \mathrm{m})$, $7.65(2 \mathrm{H}, \mathrm{d}, J 8.3 \mathrm{~Hz}), 8.03(2 \mathrm{H}, \mathrm{d}, J 8.3 \mathrm{~Hz}) . \delta_{\mathrm{C}}\left(\mathrm{CDCl}_{3}, 75.5 \mathrm{MHz}\right): 26.9,125.6(2 \mathrm{C}), 127.4$, 127.5, 128.4, 129.2 (2C), 130.4, 135.1, 136.5, 141.8, 144.3, 197.7. m/z (EI) $230\left(\mathrm{M}^{+}, 42 \%\right), 215$ (100), 187 (5), 152 (74), 76 (16), 58 (22).

1-(2'-Chlorobiphenyl-4-yl)ethanone, 2c. White solid. mp 54-55 ${ }^{\circ} \mathrm{C}$ (lit. ${ }^{1} 54-56{ }^{\circ} \mathrm{C}$, methanol). $\delta_{\mathrm{H}}$ $\left(\mathrm{CDCl}_{3}, 400 \mathrm{MHz}\right): 2.67(3 \mathrm{H}, \mathrm{s}), 7.32-7.37(3 \mathrm{H}, \mathrm{m}), 7.50-7.53(1 \mathrm{H}, \mathrm{m}), 7.57(2 \mathrm{H}, \mathrm{d}, J$ 8.2 Hz), 8.05 $\left(2 \mathrm{H}, \mathrm{d}, J\right.$ 8.2 Hz). $\delta_{\mathrm{C}}\left(\mathrm{CDCl}_{3}, 100.6 \mathrm{MHz}\right): 26.7,127.0,128.2(2 \mathrm{C}), 129.2,129.8(2 \mathrm{C}), 130.1,131.1$, 132.3, 136.2, 139.4, 144.1, 197.8. m/z (EI) $230\left(\mathrm{M}^{+}, 41 \%\right), 215$ (100), 187 (5), 152 (87), 126 (8), $107(7), 58(36)$

4-Chloro-4'-fluorobiphenyl, 2d. White solid. mp 80-81 ${ }^{\circ} \mathrm{C}$ (lit. $\left.{ }^{2} 83-88{ }^{\circ} \mathrm{C}\right) . \quad \delta_{\mathrm{H}}\left(\mathrm{CDCl}_{3}, 400\right.$ MHz): $7.12(2 \mathrm{H}, \mathrm{t}, J 8.8 \mathrm{~Hz}), 7.40(2 \mathrm{H}, \mathrm{d}, J 8.8 \mathrm{~Hz}), 7.46(2 \mathrm{H}, \mathrm{d}, J 8.8 \mathrm{~Hz}), 7.51(2 \mathrm{H}, \mathrm{dd} J 5.3,8.8$ Hz). $\delta_{\mathrm{C}}\left(\mathrm{CDCl}_{3}, 100.6 \mathrm{MHz}\right): 115.7(2 \mathrm{C}, \mathrm{d}, J 21.6 \mathrm{~Hz}), 128.2(2 \mathrm{C}), 128.5(2 \mathrm{C}, \mathrm{d}, J 8.1 \mathrm{~Hz}), 128.9$ (2C), 133.4, 136.0 (d, J 3.3 Hz), 138.6, 162.6 (d, J $246.8 \mathrm{~Hz}) . m / z(\mathrm{EI}) 206\left(\mathrm{M}^{+}, 23 \%\right), 171(33), 85$ (17), 58 (100).

4-Chlorobiphenyl, 2e. White solid. mp 70-71 ${ }^{\circ} \mathrm{C}\left(\right.$ lit. $\left.^{3} 77^{\circ} \mathrm{C}\right) . \quad \delta_{\mathrm{H}}\left(\mathrm{CDCl}_{3}, 300 \mathrm{MHz}\right)$ : 7.31-7.39 $(1 \mathrm{H}, \mathrm{m}), 7.39-7.44(2 \mathrm{H}, \mathrm{m}), 7.44-7.49(2 \mathrm{H}, \mathrm{m}), 7.50-7.61(4 \mathrm{H}, \mathrm{m}) . \delta_{\mathrm{C}}\left(\mathrm{CDCl}_{3}, 75.5 \mathrm{MHz}\right): 127.0$ (2C), 127.6, $128.4(2 \mathrm{C}), 128.9(4 \mathrm{C}), 133.4,139.7,140.0 . \mathrm{m} / \mathrm{z}(\mathrm{EI}) 188\left(\mathrm{M}^{+}, 100 \%\right), 152(55), 126$ (6), $98(3), 76(14), 58(40)$.

4-Chloro-4'-methylbiphenyl, 2f. White solid. mp 122-123 ${ }^{\circ} \mathrm{C}$ (lit. $\left.{ }^{4} 122{ }^{\circ} \mathrm{C}\right) . \delta_{\mathrm{H}}\left(\mathrm{CDCl}_{3}, 400\right.$ MHz): $2.40(3 \mathrm{H}, \mathrm{s}), 7.22-7.26(2 \mathrm{H}, \mathrm{m}), 7.39(2 \mathrm{H}, \mathrm{d}, J 8.8 \mathrm{~Hz}), 7.46(2 \mathrm{H}, \mathrm{d}, J 8.2 \mathrm{~Hz}), 7.51(2 \mathrm{H}, \mathrm{d}, J$ $8.8 \mathrm{~Hz}) . \delta_{\mathrm{C}}\left(\mathrm{CDCl}_{3}, 100.6 \mathrm{MHz}\right): 21.1,126.8(2 \mathrm{C}), 128.1(2 \mathrm{C}), 128.8,129.4,129.6(2 \mathrm{C}), 133.0$, 137.1, 137.4, 139.6. m/z (EI) $202\left(\mathrm{M}^{+}, 81 \%\right), 165$ (46), $152(20), 139(6), 82(23), 58(100)$. 
3-Chloro-4'-fluorobiphenyl, 2g. ${ }^{5}$ Colourless oil. $\delta_{\mathrm{H}}\left(\mathrm{CDCl}_{3}, 400 \mathrm{MHz}\right): 7.14(2 \mathrm{H}, \mathrm{t}, \mathrm{J} 8.8 \mathrm{~Hz})$, $7.31(1 \mathrm{H}, \mathrm{dt}, J 1.8,7.6 \mathrm{~Hz}), 7.36(1 \mathrm{H}, \mathrm{t}, J 7.6 \mathrm{~Hz}), 7.42(1 \mathrm{H}, \mathrm{dt} J 1.8,7.6 \mathrm{~Hz}), 7.48-7.56(3 \mathrm{H}, \mathrm{m}) . \delta_{\mathrm{C}}$ $\left(\mathrm{CDCl}_{3}, 100.6 \mathrm{MHz}\right): 115.8$ (2C, d, J 21.4 Hz), 125.1, 127.1, 127.3, 128.7 (2C, d, J 8.6 Hz), 130.0, 134.7, 135.9 (d, J 3.4 Hz), 142.0, 162.8 (d, J 247.8 Hz). m/z (EI) 206 (M+, 37\%), 170 (25), 85 (4), 58 (100).

3-Chlorobiphenyl, 2h. ${ }^{6}$ Colourless oil. $\delta_{\mathrm{H}}\left(\mathrm{CDCl}_{3}, 300 \mathrm{MHz}\right)$ : 7.30-7.34 (1H, m), 7.34-7.41 (2H, m), 7.42-7.50 (3H, m), 7.53-7.62 (3H, m). $\delta_{\mathrm{C}}\left(\mathrm{CDCl}_{3}, 75.5 \mathrm{MHz}\right): 125.4,127.2,127.3,127.4,127.9$ (2C), 129.0 (2C), 130.1, 134.7, 139.9, 143.1. m/z (EI) $188\left(\mathrm{M}^{+}, 84 \%\right), 152$ (54), 126 (7), 76 (23), $58(100)$

3-Chloro-4'-methylbiphenyl, 2i. White solid. mp 41-42 ${ }^{\circ} \mathrm{C}$ (lit. $\left.{ }^{7} 44-45{ }^{\circ} \mathrm{C}\right) . \quad \delta_{\mathrm{H}}\left(\mathrm{CDCl}_{3}, 400\right.$ MHz): $2.40(3 \mathrm{H}, \mathrm{s}), 7.27(2 \mathrm{H}, \mathrm{d}, J 8.2 \mathrm{~Hz}), 7.27-7.32(1 \mathrm{H}, \mathrm{m}), 7.35(1 \mathrm{H}, \mathrm{t}, J$ 7.6 Hz ), 7.42-7.50 $(1 \mathrm{H}, \mathrm{m}), 7.47(2 \mathrm{H}, \mathrm{d}, J 8.2 \mathrm{~Hz}), 7.56(1 \mathrm{H}, \mathrm{t}, J 1.8 \mathrm{~Hz}) . \delta_{\mathrm{C}}\left(\mathrm{CDCl}_{3}, 100.6 \mathrm{MHz}\right): 21.1,125.0,126.8$, 126.9 (2C), 127.0 (2C), 129.6, 129.9, 134.6, 136.9, 137.7, 142.9. m/z (EI) $202\left(\mathrm{M}^{+}, 100 \%\right), 167$ (74), 152 (33), $115(11), 82(18), 63$ (23).

2-Chlorobiphenyl, 2j. Pale yellow oil (lit. $\left.{ }^{8} 34^{\circ} \mathrm{C}\right) . \delta_{\mathrm{H}}\left(\mathrm{CDCl}_{3}, 400 \mathrm{MHz}\right): 7.30-7.37$ (2H, m), 7.38-7.40 (1H, m), 7.41-7.53 (6H, m). $\delta_{\mathrm{C}}\left(\mathrm{CDCl}_{3}, 100.6 \mathrm{MHz}\right): 126.9,127.7,128.1(2 \mathrm{C}), 128.6$, 129.5 (2C), 130.0, 131.45, 132.5, 139.5, 140.6. m/z (EI) $188\left(\mathrm{M}^{+}, 4 \%\right), 152(2), 58(100)$.

2-Chloro-4'-fluorobiphenyl, 2k. ${ }^{9}$ Pale yellow oil. $\delta_{\mathrm{H}}\left(\mathrm{CDCl}_{3}, 400 \mathrm{MHz}\right): 7.18(2 \mathrm{H}, \mathrm{t}, \mathrm{J} 8.7 \mathrm{~Hz})$, 7.32-7.38 (3H, m), $7.47(2 \mathrm{H}, \mathrm{dd}, J 5.4,8.6 \mathrm{~Hz}), 7.53(1 \mathrm{H}, \mathrm{dd}, J 2.0,6.2 \mathrm{~Hz}) . \delta_{\mathrm{C}}\left(\mathrm{CDCl}_{3}, 100.6\right.$ MHz): 114.9 (2C, d, J $21 \mathrm{~Hz}), 126.8,128.6,129.9,131.0$ (2C, d, J $8 \mathrm{~Hz}), 131.2,132.4,135.2$, 139.4, 162.3 (d, J $247 \mathrm{~Hz}) . \mathrm{m} / \mathrm{z}$ (EI) $206\left(\mathrm{M}^{+}, 43 \%\right), 188$ (16), 172 (27), 85 (10), 58 (100).

2-Chloro-4'-methoxybiphenyl, 2l. ${ }^{10}$ Pale yellow oil. $\delta_{\mathrm{H}}\left(\mathrm{CDCl}_{3}, 400 \mathrm{MHz}\right): 3.89(3 \mathrm{H}, \mathrm{s}), 7.01$ $(2 \mathrm{H}, \mathrm{d}, J 8.8 \mathrm{~Hz}), 7.26-7.38(3 \mathrm{H}, \mathrm{m}), 7.43(2 \mathrm{H}, \mathrm{d}, J 8.8 \mathrm{~Hz}), 7.71(1 \mathrm{H}, \mathrm{dd}, J 1.5,7.7 \mathrm{~Hz}) . \delta_{\mathrm{C}}\left(\mathrm{CDCl}_{3}\right.$, 
100.6 MHz): 55.3, 113.5 (2C), 126.8, 128.2, 130.0, 130.7 (2C), 131.4, 131.8, 132.6, 140.2, 159.2. m/z (EI) $218\left(\mathrm{M}^{+}, 100 \%\right), 203$ (28), 175 (38), 139 (52), 115 (10).

2-Chloro-2'-methoxybiphenyl, 2m. White solid. mp 55-56 ${ }^{\circ} \mathrm{C} . \delta_{\mathrm{H}}\left(\mathrm{CDCl}_{3}, 400 \mathrm{MHz}\right): 3.83(3 \mathrm{H}$, s), $7.04(1 \mathrm{H}, \mathrm{d}, J 8.3 \mathrm{~Hz}), 7.09(1 \mathrm{H}, \mathrm{t}, J$ 7.4 Hz), $7.26(1 \mathrm{H}, \mathrm{dd}, J 1.5,7.4 \mathrm{~Hz}), 7.30-7.38$ (3H, m), 7.41-7.46 (1H, m), 7.49-7.53 (1H, m). $\delta_{\mathrm{C}}\left(\mathrm{CDCl}_{3}, 100.6 \mathrm{MHz}\right): 55.7,111.1,120.4,126.5,128.6$, 128.65, 129.4, 129.35, 131.0, 131.8, 134.0, 137.8, 156.8. m/z (EI) $218\left(\mathrm{M}^{+}, 24 \%\right), 183(18), 168$ (39), 139 (13), 58 (100).

1-(4-Fluoro-[1,1';4',1”]terphenyl-4”-yl-ethanone, 3a. White solid. Purified by recrystallization from hexane-ethyl acetate $(4: 1)$. mp $218-219^{\circ} \mathrm{C}$. Found: C, 82.8; H, 5.25. $\mathrm{C}_{20} \mathrm{H}_{15} \mathrm{FO}$ requires C, 82.75; H, 5.2\%. v $v_{\max }\left(\mathrm{KBr}\right.$ disc)/cm $\mathrm{cm}^{-1} 3040$ (CH, w), 1681 (CO, vs), 1599 (CC), 1489 (CC), 1469 (CC), $1425(\mathrm{CC}, \mathrm{w}), 815(\mathrm{CH}, \mathrm{vs}), 716(\mathrm{CH}, \mathrm{w}) . \delta_{\mathrm{H}}\left(\mathrm{CDCl}_{3}, 400 \mathrm{MHz}\right): 2.66(3 \mathrm{H}, \mathrm{s}), 7.16(2 \mathrm{H}, \mathrm{t}, J$ $9.0 \mathrm{~Hz}), 7.61(2 \mathrm{H}, \mathrm{dd}, J$ 5.5, $9.0 \mathrm{~Hz}), 7.66(2 \mathrm{H}, \mathrm{d}, J 9.0 \mathrm{~Hz}), 7.72(2 \mathrm{H}, \mathrm{d}, J 9.0 \mathrm{~Hz}), 7.74(2 \mathrm{H}, \mathrm{d}, J$ $8.5 \mathrm{~Hz}), 8.06(2 \mathrm{H}, \mathrm{d}, J 8.5 \mathrm{~Hz}) . \delta_{\mathrm{C}}\left(\mathrm{CDCl}_{3}, 75.5 \mathrm{MHz}\right): 26.9,116.0$ (2C, d, J $\left.21 \mathrm{~Hz}\right), 127.3(2 \mathrm{C})$, 127.8 (2C), 127.9 (2C), 128.8 (2C, d, J 9.0 Hz), 129.2 (2C), 136.2, 136.8, 138.9, 140.3, 145.4, 162.9 (d, J $246 \mathrm{~Hz})$, 197.9. m/z (EI) $290\left(\mathrm{M}^{+}, 63 \%\right), 275$ (100), 246 (22), 226 (17).

1-[1,1';4',1"]terphenyl-4-yl-ethanone, 3b. White solid. Purified by recrystallization from hexane-ethyl acetate (4:1). mp 237-238 ${ }^{\circ} \mathrm{C}$ (lit. ${ }^{11} 235-237{ }^{\circ} \mathrm{C}$, dioxane). Found: C, 88.25; H, 5.9. $\mathrm{C}_{20} \mathrm{H}_{16} \mathrm{O}$ requires C, 88.2; H, 5.9\%. $\mathrm{v}_{\max }(\mathrm{KBr}$ disc $) / \mathrm{cm}^{-1} 3055(\mathrm{CH}, \mathrm{w}), 3033(\mathrm{CH}, \mathrm{w}), 1681(\mathrm{CO}$, vs), $1601(\mathrm{CC}), 1483(\mathrm{CC}), 823(\mathrm{CH}, \mathrm{s}), 766(\mathrm{CH}, \mathrm{s}), 732(\mathrm{CH}), 693(\mathrm{CH}) . \delta_{\mathrm{H}}\left(\mathrm{CDCl}_{3}, 400 \mathrm{MHz}\right)$ : $2.66(3 \mathrm{H}, \mathrm{s}), 7.39(1 \mathrm{H}, \mathrm{t}, J 7.3 \mathrm{~Hz}), 7.48(2 \mathrm{H}, \mathrm{t}, J 7.3 \mathrm{~Hz}), 7.66(2 \mathrm{H}, \mathrm{d}, J 7.3 \mathrm{~Hz}), 7.73(4 \mathrm{H}, \mathrm{s}), 7.75$ $(2 \mathrm{H}, \mathrm{d}, J 8.3 \mathrm{~Hz}), 8.07(2 \mathrm{H}, \mathrm{d}, J 8.3 \mathrm{~Hz}) . \delta_{\mathrm{C}}\left(\mathrm{CDCl}_{3}, 100.6 \mathrm{MHz}\right): 26.7,127.1$ (4C), 127.6, 127.7 (4C), 128.9 (2C), 129.0 (2C), 135.9, 138.7, 140.4, 141.1, 145.3, 197.8. m/z (EI) $272\left(\mathrm{M}^{+}, 65 \%\right), 257$ (100), 228 (29), $202(9), 152(5)$.

1-(4”-Methyl-[1,1';4',1']terphenyl-4-yl-ethanone, 3c. ${ }^{12}$ White solid. Purified by recrystallization from hexane-ethyl acetate (4:1). mp 270-271 ${ }^{\circ} \mathrm{C}$. Found: $\mathrm{C}, 88.2 ; \mathrm{H}, 6.35 . \mathrm{C}_{21} \mathrm{H}_{18} \mathrm{O}$ 
requires $\mathrm{C}, 88.1$; H, 6.35\%. $\mathrm{v}_{\max }\left(\mathrm{KBr}\right.$ disc)/ $\mathrm{cm}^{-1} 1682$ (CO, vs), 1600 (C-C), 1490 (C-C), 806 (CH, vs). $\delta_{\mathrm{H}}\left(\mathrm{CDCl}_{3}, 400 \mathrm{MHz}\right): 2.43(3 \mathrm{H}, \mathrm{s}), 2.66(3 \mathrm{H}, \mathrm{s}), 7.29(2 \mathrm{H}, \mathrm{d}, J 7.8 \mathrm{~Hz}), 7.56(2 \mathrm{H}, \mathrm{d}, J 7.8 \mathrm{~Hz})$, $7.71(4 \mathrm{H}, \mathrm{s}), 7.75(2 \mathrm{H}, \mathrm{d}, 8.3 \mathrm{~Hz}), 8.06(2 \mathrm{H}, \mathrm{d}, 8.3 \mathrm{~Hz}) . \delta_{\mathrm{C}}\left(\mathrm{CDCl}_{3}, 100.6 \mathrm{MHz}\right): 21.2,26.7,126.9$ (2C), 127.1 (2C), 127.5 (2C), 127.6 (2C), 129.0 (2C),129.6 (2C), 135.8, 137.5 (2C), 138.4, 141.1, 145.3, 197.8. m/z (EI) $286\left(\mathrm{M}^{+}, 97 \%\right), 271$ (100), 239 (7), 228 (26), 207 (15).

1-(2"-Methoxy-[1,1';4',1"]terphenyl-4-yl-ethanone, 3d. White solid. Purified by recrystallization from hexane-ethyl acetate (4:1). mp 155-156 ${ }^{\circ} \mathrm{C}$. Found: $\mathrm{C}, 83.5 ; \mathrm{H}, 5.95 . \mathrm{C}_{21} \mathrm{H}_{18} \mathrm{O}_{2}$ requires $\mathrm{C}, 83.4 ; \mathrm{H}, 6.0 \%$. $\mathrm{v}_{\max }(\mathrm{KBr} \operatorname{disc}) / \mathrm{cm}^{-1} 3053(\mathrm{CH}), 3036(\mathrm{CH}), 3011(\mathrm{CH}), 1675(\mathrm{CO}$, vs), 1604 (CC, s), 1499 (CC), 1485 (CC, s), 1462 (CC), 1438 (CC), 1423 (CC, w), 1411 (CC, w), 850 $(\mathrm{CH}), 820(\mathrm{CH}, \mathrm{s}), 803(\mathrm{CH}), 777(\mathrm{CH}, \mathrm{w}), 756(\mathrm{CH}, \mathrm{s}), 734(\mathrm{CH}), 727(\mathrm{CH}) . \delta_{\mathrm{H}}\left(\mathrm{CDCl}_{3}, 400\right.$ MHz): $2.67(3 \mathrm{H}, \mathrm{s}), 3.86(3 \mathrm{H}, \mathrm{s}), 7.04(1 \mathrm{H}, \mathrm{d}, J 8.0 \mathrm{~Hz}), 7.10(1 \mathrm{H}, \mathrm{t}, J 7.5 \mathrm{~Hz}), 7.35-7.40(2 \mathrm{H}, \mathrm{m})$, $7.67(2 \mathrm{H}, \mathrm{d}, J 8.5 \mathrm{~Hz}), 7.70(2 \mathrm{H}, \mathrm{d}, J 8.5 \mathrm{~Hz}), 7.75(2 \mathrm{H}, \mathrm{d}, J 8.3 \mathrm{~Hz}), 8.06(2 \mathrm{H}, \mathrm{d}, J 8.3 \mathrm{~Hz}) . \delta_{\mathrm{C}}$ $\left(\mathrm{CDCl}_{3}, 100.6 \mathrm{MHz}\right): 26.7,55.6,111.3,121.0,126,9$ (2C), 127.2 (2C), 129.0 (2C), 129.9, 130.2 (2C), 130.8 (2C), 135.8, 138.3, 138.7, 145.6, 156.5, 197.8. m/z (EI) $302\left(\mathrm{M}^{+}, 92 \%\right), 287$ (100), 244 (17), $226(9), 215$ (17).

1-(4"-Methoxy-[1,1';4',1"]terphenyl-4-yl-ethanone, 3e. White solid. Purified by recrystallization from hexane-ethyl acetate (4:1). mp 286-287 ${ }^{\circ} \mathrm{C}$. Found: $\mathrm{C}, 83.5 ; \mathrm{H}, 6.0 . \mathrm{C}_{21} \mathrm{H}_{18} \mathrm{O}_{2}$ requires $\mathrm{C}, 83.4 ; \mathrm{H}, 6.0 \%$. $\mathrm{v}_{\max }(\mathrm{KBr}$ disc $) / \mathrm{cm}^{-1} 3036(\mathrm{CH}), 3001(\mathrm{CH}), 1678(\mathrm{CO}, \mathrm{vs}), 1600(\mathrm{CC}$, s), 1489 (CC), 1464 (CC), 1445 (CC), 1427 (CC), 813 (CH, vs), $716(\mathrm{CH}) . \delta_{\mathrm{H}}\left(\mathrm{CDCl}_{3}, 400 \mathrm{MHz}\right)$ : $2.66(3 \mathrm{H}, \mathrm{s}), 3.88(3 \mathrm{H}, \mathrm{s}), 7.02(2 \mathrm{H}, \mathrm{d}, J 8.8 \mathrm{~Hz}), 7.60(2 \mathrm{H}, \mathrm{d}, J 8.8 \mathrm{~Hz}), 7.67(2 \mathrm{H}, \mathrm{d}, J 8.6 \mathrm{~Hz}), 7.71$ (2H, d, $J 8.6 \mathrm{~Hz}), 7.74(2 \mathrm{H}, \mathrm{d}, J 8.6 \mathrm{~Hz}), 8.06(2 \mathrm{H}, \mathrm{d}, J 8.6 \mathrm{~Hz}) . \delta_{\mathrm{C}}\left(\mathrm{CDCl}_{3}, 75.5 \mathrm{MHz}\right): 26.6,55.6$, 114.5 (2C), 127.0 (2C), 127.3 (2C), 127.6 (2C), 128.1 (2C), 128.9 (2C), 132.8, 135.6, 138.0, 140.6, 145.2, 159.3, 197.7. m/z (EI) $302\left(\mathrm{M}^{+}, 100 \%\right), 287$ (75), 259 (14), 244 (10), 215 (29), 189 (9). 
4-Fluoro-[1,1';4',1”]terphenyl, 3f. White solid. Purified by recrystallization from hexane. mp 216-217 (lit. ${ }^{13} 214-215^{\circ} \mathrm{C}$ ). Found: C, 87.15; H, 5.3. $\mathrm{C}_{18} \mathrm{H}_{13} \mathrm{~F}$ requires $\mathrm{C}, 87.1 ; \mathrm{H}, 5.3 \%$. $\mathrm{v}_{\max }(\mathrm{KBr}$ disc) $/ \mathrm{cm}^{-1} 3056(\mathrm{CH}), 3034(\mathrm{CH}), 1598$ (CC), 1485 (CC, s), 1449 (CC, w), 1416 (CC, w), 824 (CH, s), $764(\mathrm{CH}, \mathrm{s}), 725(\mathrm{CH}, \mathrm{w}), 691(\mathrm{CH}) . \delta_{\mathrm{H}}\left(\mathrm{CDCl}_{3}, 400 \mathrm{MHz}\right): 7.16(2 \mathrm{H}, \mathrm{t}, J 8.5 \mathrm{~Hz}), 7.37(1 \mathrm{H}, \mathrm{tt}$, $J$ 1.0, 7.0 Hz), $7.47(2 \mathrm{H}, \mathrm{t}, J 8.0 \mathrm{~Hz}), 7.58-7.70(8 \mathrm{H}, \mathrm{m}) . \delta_{\mathrm{C}}\left(\mathrm{CDCl}_{3}, 100.6 \mathrm{MHz}\right): 115.7(2 \mathrm{C}, \mathrm{d}, J 21$ Hz), 127.1 (2C), 127.3 (2C), 127.4, 127.6 (2C), 128.6 (2C, d, J 7.5 Hz), 128.9 (2C), 136.8, 139.1, 140.1, 140.6, 162.5 (d, J $246 \mathrm{~Hz}) . \mathrm{m} / \mathrm{z}$ (EI) $248\left(\mathrm{M}^{+}, 100 \%\right), 244$ (6), 226 (6).

4-Fluoro-4”-methyl-[1,1';4',1”]terphenyl, 3g. White solid. Purified by recrystallization from hexane. mp 231-232 ${ }^{\circ} \mathrm{C}$. Found: $\mathrm{C}, 86.9 ; \mathrm{H}, 5.8 . \mathrm{C}_{19} \mathrm{H}_{15} \mathrm{~F}$ requires $\mathrm{C}, 87.0 ; \mathrm{H}, 5.8 \%$. $\mathrm{v}_{\max }(\mathrm{KBr}$ disc)/cm $\mathrm{cm}^{-1} 3034$ (CH, w), 1602 (CC), 1491 (CC, s), 1469 (CC, w), $834(\mathrm{CH}, \mathrm{w}), 808$ (CH, s), 735 $(\mathrm{CH}, \mathrm{w}) . \delta_{\mathrm{H}}\left(\mathrm{CDCl}_{3}, 400 \mathrm{MHz}\right): 2.42(3 \mathrm{H}, \mathrm{s}), 7.15(2 \mathrm{H}, \mathrm{t}, J 8.7 \mathrm{~Hz}), 7.28(2 \mathrm{H}, \mathrm{d}, J 8.7 \mathrm{~Hz}), 7.55$ $(2 \mathrm{H}, \mathrm{d}, J 8.0 \mathrm{~Hz}), 7.58-7.63(4 \mathrm{H}, \mathrm{m}), 7.66(2 \mathrm{H}, \mathrm{d}, J 8.0 \mathrm{~Hz}) . \delta_{\mathrm{C}}\left(\mathrm{CDCl}_{3}, 100.6 \mathrm{MHz}\right): 21.2,115.7$ (2C, d, J $21 \mathrm{~Hz}), 126.9$ (2C), 127.3 (4C), 128.6 (2C, d, J 8.7 Hz), 129.6 (2C), 136.9, 137.3, 137.7, $138.9,140.1,162.5$ (d, J $246 \mathrm{~Hz}) . \mathrm{m} / \mathrm{z}$ (EI) $262\left(\mathrm{M}^{+}, 100 \%\right), 246(8)$.

4-Fluoro-2"-methoxy-[1,1';4',1"]terphenyl, 3h. White solid. Purified by column chromatography, $R_{f} 0.4$ (hexane- $\mathrm{CH}_{2} \mathrm{Cl}_{2}, 4: 1$ ). mp 125-126 ${ }^{\circ} \mathrm{C}$. Found: C, 82.05; H, 5.45. $\mathrm{C}_{19} \mathrm{H}_{15} \mathrm{FO}$ requires C, 82.0; H, 5.4\%. $v_{\max }\left(\mathrm{KBr}\right.$ disc) $/ \mathrm{cm}^{-1} 3067(\mathrm{CH}, \mathrm{w}), 3039(\mathrm{CH}, \mathrm{w}), 3027(\mathrm{CH}, \mathrm{w}), 3007$ (CH, w), 1597 (CC), 1483 (CC, s), 1460 (CC), 1434 (CC), 1415 (CC, w), 854 (CH, w), 823 (CH, s), $799(\mathrm{CH}), 763(\mathrm{CH}, \mathrm{s}) . \delta_{\mathrm{H}}\left(\mathrm{CDCl}_{3}, 400 \mathrm{MHz}\right): 3.86(3 \mathrm{H}, \mathrm{s}), 7.08(1 \mathrm{H}, \mathrm{d}, J 8.0 \mathrm{~Hz}), 7.13(1 \mathrm{H}, \mathrm{t}, J$ $7.6 \mathrm{~Hz}), 7.21(2 \mathrm{H}, \mathrm{t}, J 8.5 \mathrm{~Hz}), 7.41(1 \mathrm{H}, \mathrm{dd}, J 2.0,7.6 \mathrm{~Hz}), 7.45(1 \mathrm{H}, \mathrm{ddd}, J 2.0,7.6,8.0 \mathrm{~Hz}), 7.65-$ $7.71(6 \mathrm{H}, \mathrm{m}) . \delta_{\mathrm{C}}\left(\mathrm{CDCl}_{3}, 100.6 \mathrm{MHz}\right): 55.5,111.3,115.7(2 \mathrm{C}, \mathrm{d}, \mathrm{J} 21 \mathrm{~Hz}), 120.9,126.7$ (2C), 128.6 (2C, d, J 6.9 Hz), 128.9, 130.0 (2C), 130.1, 130.9, 137.2 (d, J 3.1 Hz), 137.6, 138.9, 156.5, 162.4 (d, $J 246 \mathrm{~Hz}) . \mathrm{m} / \mathrm{z}(\mathrm{EI}) 278\left(\mathrm{M}^{+}, 100 \%\right), 263$ (17), 245 (15), 233 (17), 215 (27). 
4”-Methyl-[1,1';4',1”]terphenyl, 3i. White solid. Purified by recrystallization from hexane. mp 206-208 ${ }^{\circ} \mathrm{C}$ (lit. ${ }^{14} 209-210{ }^{\circ} \mathrm{C}$ ). Found: C, 93.5; H, 6.65. $\mathrm{C}_{19} \mathrm{H}_{16}$ requires $\mathrm{C}, 93.4 ; \mathrm{H}, 6.6 \%$. v $\max$ (KBr disc)/cm 3048 (CH, w), 3026 (CH, w), 1596 (CC, w), 1482 (CC, s), 1448 (CC), 1409 (CC), $851(\mathrm{CH}), 814(\mathrm{CH}, \mathrm{s}), 763(\mathrm{CH}, \mathrm{s}), 728(\mathrm{CH}), 695(\mathrm{CH}), 666(\mathrm{CH}, \mathrm{w}) . \delta_{\mathrm{H}}\left(\mathrm{CDCl}_{3}, 400 \mathrm{MHz}\right): 2.43$ $(3 \mathrm{H}, \mathrm{s}), 7.28(2 \mathrm{H}, \mathrm{d}, J 8.0 \mathrm{~Hz}), 7.37(1 \mathrm{H}, \mathrm{t}, J 8.0 \mathrm{~Hz}), 7.47(2 \mathrm{H}, \mathrm{t}, J 7.7 \mathrm{~Hz}), 7.56(2 \mathrm{H}, \mathrm{d}, J 8.0 \mathrm{~Hz})$, $7.66(2 \mathrm{H}, \mathrm{d}, J 7.7 \mathrm{~Hz}), 7.68(4 \mathrm{H}, \mathrm{s}) . \delta_{\mathrm{C}}\left(\mathrm{CDCl}_{3}, 100.6 \mathrm{MHz}\right): 21.1,126.9(2 \mathrm{C}), 127.1(2 \mathrm{C}), 127.3$ (3C), 127.5 (2C), 128.8 (2C), $129.6(2 \mathrm{C}), 137.2,137.8,139.9,140.1,140.8 . \mathrm{m} / \mathrm{z}(\mathrm{EI}) 244\left(\mathrm{M}^{+}\right.$, 100\%), $228(11), 202(6), 165(12)$.

2”-Methoxy-[1,1';4',1”]terphenyl, 3j. White solid. Purified by column chromatography, $R_{f} 0.35$ (hexane- $\mathrm{CH}_{2} \mathrm{Cl}_{2}, 7: 3$ ). mp $118-119^{\circ} \mathrm{C}$ (lit. ${ }^{15} 118-119{ }^{\circ} \mathrm{C}$ ). Found: $\mathrm{C}, 87.75 ; \mathrm{H}, 6.2 . \mathrm{C}_{19} \mathrm{H}_{16} \mathrm{O}$ requires C, 87.65; H, 6.2\%. U $v_{\max }\left(\mathrm{KBr}\right.$ disc) $/ \mathrm{cm}^{-1} 3074(\mathrm{CH}), 3053(\mathrm{CH}), 3025(\mathrm{CH}), 3000(\mathrm{CH}), 1598(\mathrm{CC})$, $1480(\mathrm{CC}, \mathrm{s}), 1462,839(\mathrm{CH}), 802(\mathrm{CH}, \mathrm{w}), 759(\mathrm{CH}, \mathrm{vs}), 745(\mathrm{CH}, \mathrm{vs}), 703(\mathrm{CH}), 687(\mathrm{CH}) . \delta_{\mathrm{H}}$ $\left(\mathrm{CDCl}_{3}, 400 \mathrm{MHz}\right): 3.86(3 \mathrm{H}, \mathrm{s}), 7.02(1 \mathrm{H}, \mathrm{d}, J 8.3 \mathrm{~Hz}), 7.06(1 \mathrm{H}, \mathrm{td}, J 1.0,7.3 \mathrm{~Hz}), 7.33-7.39(3 \mathrm{H}$, m), $7.46(2 \mathrm{H}, \mathrm{t}, J 7.8 \mathrm{~Hz}), 7.62-7.84(6 \mathrm{H}, \mathrm{m}) . \delta_{\mathrm{C}}\left(\mathrm{CDCl}_{3}, 100.6 \mathrm{MHz}\right): 55.6,111.3,120.9,126.8$ (2C), 127.1 (2C), 127.2, 128.7, 128.8 (2C), 130.0 (2C), 130.2, 130.9, 137.6, 139.8, 141.1, 156.6. m/z (EI) $260\left(\mathrm{M}^{+}, 100 \%\right), 245$ (12), 227 (22), 215 (14), 202 (11).

2"-Methoxy-4-methyl-[1,1';4',1"]terphenyl, 3k. White solid. Purified by column chromatography, $R_{f} 0.4$ (hexane- $\mathrm{CH}_{2} \mathrm{Cl}_{2}$, 7:3). mp 106-107 ${ }^{\circ} \mathrm{C}$. Found: $\mathrm{C}, 87.6 ; \mathrm{H}, 6.7 . \mathrm{C}_{20} \mathrm{H}_{18} \mathrm{O}$ requires $\mathrm{C}, 87.55 ; \mathrm{H}, 6.6 \%$. $\mathrm{v}_{\max }(\mathrm{KBr}$ disc $) / \mathrm{cm}^{-1} 3078(\mathrm{CH}), 3045(\mathrm{CH}), 3020(\mathrm{CH}), 1596(\mathrm{CC})$, $1501(\mathrm{CC}), 1481$ (CC, s), 1457 (CC, s), 1433 (CC), $855(\mathrm{CH}), 807$ (CH, s), $754(\mathrm{CH}, \mathrm{s}) . \delta_{\mathrm{H}}\left(\mathrm{CDCl}_{3}\right.$, $400 \mathrm{MHz}): 2.41(3 \mathrm{H}, \mathrm{s}), 3.85(3 \mathrm{H}, \mathrm{s}), 7.02(1 \mathrm{H}, \mathrm{d}, J 8.3 \mathrm{~Hz}), 7.06(1 \mathrm{H}, \mathrm{td}, J 1.0,7.5 \mathrm{~Hz}), 7.28(2 \mathrm{H}$, d, J 7.5 Hz), $7.35(1 \mathrm{H}, \mathrm{td}, J 2.0,8.3 \mathrm{~Hz}), 7.38(1 \mathrm{H}, \mathrm{dd}, J 2.0,7.5 \mathrm{~Hz}), 7.56(2 \mathrm{H}, \mathrm{d}, J 8.3 \mathrm{~Hz}), 7.60-$ $7.65(4 \mathrm{H}, \mathrm{m}) . \delta_{\mathrm{C}}\left(\mathrm{CDCl}_{3}, 100.6 \mathrm{MHz}\right): 21.1,55.6,111.2,120.9,126.6(2 \mathrm{C}), 127.0(2 \mathrm{C}), 128.7$, $129.6(2 \mathrm{C}), 129.9$ (2C), 130.3, 130.9, 137.0, 137.3, 138.2, 139.7, 156.6. m/z (EI) $274\left(\mathrm{M}^{+}, 100 \%\right)$, 258 (13), 244 (23), 241 (13), 215 (14). 
1-(4-Fluoro-[1,1';3',1”]terphenyl-4”-yl)-ethanone, 4a. White solid. Purified by column chromatography, $R_{f} 0.3\left(\mathrm{CH}_{2} \mathrm{Cl}_{2}\right)$. mp 103-104 ${ }^{\circ} \mathrm{C}$. Found: C, 82.8; H, 5.2. $\mathrm{C}_{20} \mathrm{H}_{15} \mathrm{FO}$ requires $\mathrm{C}$,

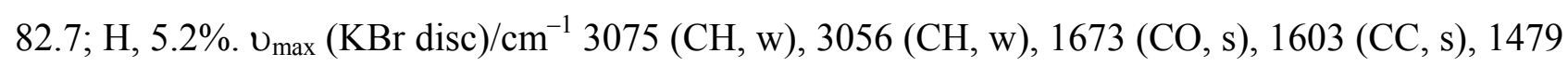
(CC, s), 1436 (CC, w), 1423 (CC, w), 1407 (CC), $842(\mathrm{CH}), 827(\mathrm{CH}), 813(\mathrm{CH}), 792$ (CH, s). $\delta_{\mathrm{H}}\left(\mathrm{CDCl}_{3}, 400 \mathrm{MHz}\right): 2.66(3 \mathrm{H}, \mathrm{s}), 7.17(2 \mathrm{H}, \mathrm{t}, \mathrm{J} 9.0 \mathrm{~Hz}), 7.53-7.63(5 \mathrm{H}, \mathrm{m}), 7.47(2 \mathrm{H}, \mathrm{d}, \mathrm{J} \mathrm{9.0} \mathrm{Hz})$, $7.78(1 \mathrm{H}, \mathrm{t}, J 2 \mathrm{~Hz}), 8.07(2 \mathrm{H}, \mathrm{d}, J 9.0 \mathrm{~Hz}) . \delta_{\mathrm{C}}\left(\mathrm{CDCl}_{3}, 100.6 \mathrm{MHz}\right): 26.7,115.8(2 \mathrm{C}, \mathrm{d}, J 21 \mathrm{~Hz})$, 126.1, 126.2, 127.0, 127.4 (2C), 128.8 (2C, d, J 6.9 Hz), 129.0 (2C), 129.5, 136.0, 137.0, 140.6, 141.1, 145.6, 162.7 (d, J 246 Hz), 197.8. m/z (EI) 290 (M+, 52\%), 275 (100), 246 (21), 226 (16).

1-[1,1';3’,1”]Terphenyl-4"-yl-ethanone, 4b. White solid. Purified by column chromatography, $R_{f} 0.12$ (hexane- $\mathrm{CH}_{2} \mathrm{Cl}_{2}, 3: 2$ ). $\mathrm{mp} 103-104{ }^{\circ} \mathrm{C}$ (lit. ${ }^{1}$ 103-104 ${ }^{\circ} \mathrm{C}$, ethanol). Found: C, 88.3; H, 5.9. $\mathrm{C}_{20} \mathrm{H}_{16} \mathrm{O}$ requires C, 88.2; H, 5.9\%. $\mathrm{v}_{\max }(\mathrm{KBr}$ disc $) / \mathrm{cm}^{-1} 3061(\mathrm{CH}, \mathrm{w}), 3034(\mathrm{CH}, \mathrm{w}), 1680(\mathrm{CO}$, vs), 1600 (CC, s), 1475 (CC), 1452 (CC), 1421 (CC), 852 (CH), $842(\mathrm{CH}), 831(\mathrm{CH}), 808(\mathrm{CH})$, $797(\mathrm{CH}), 760(\mathrm{CH}, \mathrm{s}), 700(\mathrm{CH}) . \delta_{\mathrm{H}}\left(\mathrm{CDCl}_{3}, 400 \mathrm{MHz}\right): 2.66(3 \mathrm{H}, \mathrm{s}), 7.40(1 \mathrm{H}, \mathrm{tt}, J$ 1.0, $7.3 \mathrm{~Hz})$, $7.49(2 \mathrm{H}, \mathrm{t}, J 7.8 \mathrm{~Hz}), 7.56(1 \mathrm{H}, \mathrm{t}, J 7.5 \mathrm{~Hz}), 7.61-7.67(4 \mathrm{H}, \mathrm{m}), 7.76(2 \mathrm{H}, \mathrm{d}, J 8.3 \mathrm{~Hz}), 7.84(1 \mathrm{H}, \mathrm{br}$ s), $8.07(2 \mathrm{H}, \mathrm{d}, J 8.3 \mathrm{~Hz}) . \delta_{\mathrm{C}}\left(\mathrm{CDCl}_{3}, 100.6 \mathrm{MHz}\right): 26.7,126.2(2 \mathrm{C}), 127.1,127.3(2 \mathrm{C}), 127,4(2 \mathrm{C})$, 127.6, 128.9 (2C), 129.0 (2C), 129.4, 136.0, 140.5, 140.9, 142.1, 145.8, 197.8. m/z (EI) $272\left(\mathrm{M}^{+}\right.$, 52\%), 257 (100), $228(30), 202(11), 152(6)$.

1-(4”-Methyl-[1,1';3',1"]terphenyl-4-yl-ethanone, 4c. White solid. Purified by column chromatography, $R_{f} 0.1$ (hexane- $\mathrm{CH}_{2} \mathrm{Cl}_{2}, 3: 2$ ). mp 143-144 ${ }^{\circ} \mathrm{C}$. Found: $\mathrm{C}, 88.2 ; \mathrm{H}, 6.4 . \mathrm{C}_{21} \mathrm{H}_{18} \mathrm{O}$ requires $\mathrm{C}, 88.1 ; \mathrm{H}, 6.3 \%$. v $\max (\mathrm{KBr}$ disc $) / \mathrm{cm}^{-1} 3061(\mathrm{CH}, \mathrm{w}), 3031(\mathrm{CH}, \mathrm{w}), 1687(\mathrm{CO}, \mathrm{vs}), 1602$ (CC), 1475 (CC, w), 1437 (CC, w), 1423 (CC, w), 847 (CH), 829 (CH), 788 (CH, vs), 739 (CH, w), $724(\mathrm{CH}, \mathrm{w}), 695(\mathrm{CH}) . \delta_{\mathrm{H}}\left(\mathrm{CDCl}_{3}, 400 \mathrm{MHz}\right): 2.43(3 \mathrm{H}, \mathrm{s}), 2.66(3 \mathrm{H}, \mathrm{s}), 7.30(2 \mathrm{H}, \mathrm{d}, J 7.0 \mathrm{~Hz})$, 7.52-7.65 (5H, m), $7.57(2 \mathrm{H}, \mathrm{d}, J 8.3 \mathrm{~Hz}), 7.84(1 \mathrm{H}, \mathrm{s}), 8.07(2 \mathrm{H}, \mathrm{d}, J 8.3 \mathrm{~Hz}) . \delta_{\mathrm{C}}\left(\mathrm{CDCl}_{3}, 100.6\right.$ $\mathrm{MHz}): \quad 21.2,26.7,126.0,126,1,126.9,127.1$ (2C), 127.4 (2C), 129.0 (2C), 129.4, 129.6 (2C), 
136.0, 137.5, 138.0, 140.4, 142.0, 145.9, 197.8. m/z (EI) $286\left(\mathrm{M}^{+}, 62 \%\right), 271$ (100), 239 (7), 228 (27).

1-(2”-Methoxy-[1,1';3’-1”]terphenyl-4-yl)-ethanone, 4d. White solid. Purified by column chromatography, $R_{f} 0.3\left(\mathrm{CH}_{2} \mathrm{Cl}_{2}\right)$. mp 93-94 ${ }^{\circ} \mathrm{C}$. Found: C, 83.5; H, 6.1. $\mathrm{C}_{21} \mathrm{H}_{18} \mathrm{O}_{2}$ requires C, 83.4; H, 6.0\%. $v_{\max }\left(\mathrm{KBr}\right.$ disc) $/ \mathrm{cm}^{-1} 3058(\mathrm{CH}), 3039(\mathrm{CH}), 3002(\mathrm{CH}), 1677(\mathrm{CO}, \mathrm{vs}), 1603(\mathrm{CC}, \mathrm{s})$, $1497(\mathrm{CC}), 1470(\mathrm{CC}), 1421(\mathrm{CC}), 852(\mathrm{CH}), 830(\mathrm{CH}), 793(\mathrm{CH}), 743(\mathrm{CH}), 728(\mathrm{CH}), 703(\mathrm{CH})$. $\delta_{\mathrm{H}}\left(\mathrm{CDCl}_{3}, 400 \mathrm{MHz}\right): 2.67(3 \mathrm{H}, \mathrm{s}), 3.87(3 \mathrm{H}, \mathrm{s}), 7.07(1 \mathrm{H}, \mathrm{d}, J 8.5 \mathrm{~Hz}), 7.11(1 \mathrm{H}, \mathrm{t}, J 7.0 \mathrm{~Hz}), 7.40$ $(1 \mathrm{H}, \mathrm{d}, J 7.8 \mathrm{~Hz}), 7.43(1 \mathrm{H}, \mathrm{d}, J 7.8 \mathrm{~Hz}), 7.55(1 \mathrm{H}, \mathrm{t}, J 7.8 \mathrm{~Hz}), 7.64(2 \mathrm{H}, \mathrm{t}, J 7.8 \mathrm{~Hz}), 7.77$ (2H, d, $J$ $8.0 \mathrm{~Hz}), 7.86(1 \mathrm{H}, \mathrm{s}), 8.08(2 \mathrm{H}, \mathrm{d}, J 8.0 \mathrm{~Hz}) . \delta_{\mathrm{C}}\left(\mathrm{CDCl}_{3}, 100.6 \mathrm{MHz}\right): 26.7,55.6,111.3,121.0$, $125.9,127.4$ (2C), 128.6, 128.7, 129.0, 129.1 (2C), 129.6, 130.3, 130.9, 135.9, 139.4, 139.7, 146.0, 156.5, 197.8. m/z (EI) $302\left(\mathrm{M}^{+}, 73 \%\right), 287$ (100), 244 (20), $226(10), 215$ (17), 213 (7).

1-(4-Methoxy-[1,1';3'-1”]terphenyl-4-yl)-ethanone, 4e. White solid. Purified by recrystallization from hexane. $\mathrm{mp} 157-158^{\circ} \mathrm{C}$. Found: $\mathrm{C}, 83.5 ; \mathrm{H}, 6.05 . \mathrm{C}_{21} \mathrm{H}_{18} \mathrm{O}_{2}$ requires $\mathrm{C}, 83.4$; H, 6.0\%. $v_{\max }(\mathrm{KBr}$ disc $) / \mathrm{cm}^{-1} 3059(\mathrm{CH}), 3035(\mathrm{CH}), 1688(\mathrm{CO}, \mathrm{vs}), 1604(\mathrm{CC}), 1474(\mathrm{CC}), 1460$ (CC), $1440(\mathrm{CC}), 840(\mathrm{CH}), 820(\mathrm{CH}, w), 789(\mathrm{CH}), 742(\mathrm{CH}, \mathrm{w}), 724(\mathrm{CH}, \mathrm{w}), 696(\mathrm{CH}) . \delta_{\mathrm{H}}$ $\left(\mathrm{CDCl}_{3}, 400 \mathrm{MHz}\right): 2.66(3 \mathrm{H}, \mathrm{s}), 3.88(3 \mathrm{H}, \mathrm{s}), 7.02(2 \mathrm{H}, \mathrm{d}, J 8.3 \mathrm{~Hz}), 7.51-7.61(5 \mathrm{H}, \mathrm{m}), 7.75(2 \mathrm{H}$, d, J $8.3 \mathrm{~Hz}), 7.80(1 \mathrm{H}, \mathrm{t}, J 1.0 \mathrm{~Hz}), 8.06(2 \mathrm{H}, \mathrm{d}, J 8.3 \mathrm{~Hz}) . \delta_{\mathrm{C}}\left(\mathrm{CDCl}_{3}, 100.6 \mathrm{MHz}\right): 26.7,55.4$, 114.3 (2C), 125.6, 125.8, 126.7, 127.4 (2C), 128.3 (2C), 129.0 (2C), 129.4, 133.4, 135.9, 140.4, 141.6, 145.9, 159.4, 197.8. m/z (EI) 302 (M+100\%), 287 (98), 259 (12), 244 (11), 215 (38).

4-Fluoro-[1,1';3',1”]terphenyl, 4f. Colourless solid. Purified by column chromatography, $R_{f} 0.3$ (hexane). mp 89-90 ${ }^{\circ} \mathrm{C}$. Found: $\mathrm{C}, 87.15 ; \mathrm{H}$, 5.3. $\mathrm{C}_{18} \mathrm{H}_{13} \mathrm{~F}$ requires $\mathrm{C}, 87.1 ; \mathrm{H}, 5.3 \%$. $\mathrm{v}_{\max }(\mathrm{KBr}$ disc) $/ \mathrm{cm}^{-1} 3060(\mathrm{CH}), 3035(\mathrm{CH}), 1601$ (CC), 1472 (CC), 1455 (CC), 1423 (CC), 886 (CH, w), 842 $(\mathrm{CH}), 825(\mathrm{CH}), 797(\mathrm{CH}), 783(\mathrm{CH}), 759(\mathrm{CH}, \mathrm{vs}), 719(\mathrm{CH}), 700(\mathrm{CH}) . \delta_{\mathrm{H}}\left(\mathrm{CDCl}_{3}, 400 \mathrm{MHz}\right)$ : $7.16(2 \mathrm{H}, \mathrm{t}, J 8.6 \mathrm{~Hz}), 7.39(1 \mathrm{H}, \mathrm{t}, J 7.3 \mathrm{~Hz}), 7.48(2 \mathrm{H}, \mathrm{d}, J$ 7.8 Hz), 7.52-7.54 (2H, m), 7.58-7.63 
(3H, m), $7.66(2 \mathrm{H}, \mathrm{d}, J 7.8 \mathrm{~Hz}), 7.77(1 \mathrm{H}, \mathrm{s}) . \delta_{\mathrm{C}}\left(\mathrm{CDCl}_{3}, 100.6 \mathrm{MHz}\right): 115.7(2 \mathrm{C}, \mathrm{d}, J 21 \mathrm{~Hz}), 126.0$, 126.1, 126.2, 127.3 (2C), 127.5, 128.8(2C, d, J 8.6 Hz), 128.9 (2C), 129.3, 137.3, 140.8, 141.1, 141.9, 162.6 (d, J $246 \mathrm{~Hz}) . \mathrm{m} / \mathrm{z}(\mathrm{EI}) 248\left(\mathrm{M}^{+}, 100 \%\right), 233$ (7), $226(10)$.

4-Fluoro-4"-methyl-[1,1';3',1"]terphenyl, 4g. Colourless solid. Purified by column chromatography, $R_{f} 0.4$ (hexane- $\mathrm{CH}_{2} \mathrm{Cl}_{2}, 9: 1$ ). mp 95-96 ${ }^{\circ} \mathrm{C}$. Found: $\mathrm{C}, 87.1 ; \mathrm{H}, 5.8 . \mathrm{C}_{19} \mathrm{H}_{15} \mathrm{~F}$ requires $\mathrm{C}, 87.0 ; \mathrm{H}, 5.75 \%$. $v_{\max }(\mathrm{KBr} \mathrm{disc}) / \mathrm{cm}^{-1} 3091(\mathrm{CH}), 3061(\mathrm{CH}), 3031(\mathrm{CH}), 1602(\mathrm{CC})$, $1477(\mathrm{CC}), 841(\mathrm{CH}), 824(\mathrm{CH}), 787(\mathrm{CH}, \mathrm{vs}), 697(\mathrm{CH}) . \delta_{\mathrm{H}}\left(\mathrm{CDCl}_{3}, 400 \mathrm{MHz}\right): 2.42(3 \mathrm{H}, \mathrm{s}), 7.15$ $(2 \mathrm{H}, \mathrm{t}, J 8.8 \mathrm{~Hz}), 7.28(2 \mathrm{H}, \mathrm{d}, J 8.8 \mathrm{~Hz}), 7.49-7.62(7 \mathrm{H}, \mathrm{m}), 7.74(1 \mathrm{H}, \mathrm{s}) . \delta_{\mathrm{C}}\left(\mathrm{CDCl}_{3}, 100.6 \mathrm{MHz}\right)$ : 21.2, 115.7 (2C, d, J $22 \mathrm{~Hz}), 125.7,125.8,126.0,127.1$ (2C), 128.8 (2C, d, J $8 \mathrm{~Hz}), 129.3,129.6$ (2C), 137.3 (2C), 138.2, 140.8, 141.8, 162.5 (d, J $247 \mathrm{~Hz}) . \mathrm{m} / \mathrm{z}$ (EI) $262\left(\mathrm{M}^{+}, 100 \%\right), 246$ (10), 165 (12).

4-Fluoro-2"-methoxy-[1,1';3',1"]terphenyl, 4h. White solid. Purified by column chromatography, $R_{f} 0.3$ (hexane- $\mathrm{CH}_{2} \mathrm{Cl}_{2}, 4: 1$ ). mp 58-59 ${ }^{\circ} \mathrm{C}$. Found: $\mathrm{C}, 81.9 ; \mathrm{H}, 5.45 . \mathrm{C}_{19} \mathrm{H}_{15} \mathrm{FO}$ requires $\mathrm{C}, 82.0 ; \mathrm{H}, 5.45 \%$. $\mathrm{v}_{\max }(\mathrm{KBr} \operatorname{disc}) / \mathrm{cm}^{-1} 3052(\mathrm{CH}), 3035(\mathrm{CH}), 3009(\mathrm{CH}), 1601(\mathrm{CC})$, 1470 (CC, vs), 1443 (CC), $1421(\mathrm{CC}), 843(\mathrm{CH}, \mathrm{s}), 826(\mathrm{CH}), 794(\mathrm{CH}), 765$ (CH, vs), $743(\mathrm{CH})$, $720(\mathrm{CH}, \mathrm{w}), 702(\mathrm{CH}) . \delta_{\mathrm{H}}\left(\mathrm{CDCl}_{3}, 400 \mathrm{MHz}\right): 3.84(3 \mathrm{H}, \mathrm{s}), 7.02(1 \mathrm{H}, \mathrm{d}, J 7.7 \mathrm{~Hz}), 7.06(1 \mathrm{H}, \mathrm{td}, J$ 1.0, $7.3 \mathrm{~Hz}), 7.13(2 \mathrm{H}, \mathrm{t}, J 8.8 \mathrm{~Hz}), 7.34-7.39(2 \mathrm{H}, \mathrm{m}), 7.57-7.61(2 \mathrm{H}, \mathrm{m}), 7.70(1 \mathrm{H}$, br s$) . \delta_{\mathrm{C}}$ $\left(\mathrm{CDCl}_{3}, 100.6 \mathrm{MHz}\right): 55.6,111.3,115.7(2 \mathrm{C}, \mathrm{d}, J 21 \mathrm{~Hz}), 121.0,125.7,128.4,128.6(2 \mathrm{C}, \mathrm{d}, J 7$ Hz), 128.8, 128.9 (2C), 130.5, 131.0, 137.6, 139.1, 140.1, 156.6, 162.4 (d, J $244 \mathrm{~Hz}) . \mathrm{m} / \mathrm{z}$ (EI) 278 $\left(\mathrm{M}^{+}, 100 \%\right), 263(13), 243(12), 232(20), 215(20), 160(18)$.

4-Methyl-[1,1';3',1"]terphenyl, 4i. ${ }^{6}$ Purified by column chromatography, $R_{f} 0.25$ (hexane). mp 77-78 ${ }^{\circ} \mathrm{C}$. Found: $\mathrm{C}, 93.35 ; \mathrm{H}, 6.7 . \mathrm{C}_{19} \mathrm{H}_{16}$ requires $\mathrm{C}, 93.4 ; \mathrm{H}, 6.6 \mathrm{v}_{\max }(\mathrm{KBr} \mathrm{disc}) / \mathrm{cm}^{-1} 3057(\mathrm{CH})$, 3028 (CH), 1598 (CC), 1496 (CC), 1474 (CC), 1453 (CC), 1419 (CC), 887 (CH, w), 826 (CH), 795 (CH), $757(\mathrm{CH}, \mathrm{vs}), 699(\mathrm{CH}) . \delta_{\mathrm{H}}\left(\mathrm{CDCl}_{3}, 400 \mathrm{MHz}\right): 2.42(3 \mathrm{H}, \mathrm{s}), 7.28(2 \mathrm{H}, \mathrm{d}, J 8.8 \mathrm{~Hz}), 7.37$ 
$(1 \mathrm{H}, \mathrm{tt}, J 1.0,7.5 \mathrm{~Hz}), 7.47(2 \mathrm{H}, \mathrm{t}, J 7.3 \mathrm{~Hz}), 7.51(1 \mathrm{H}, \mathrm{d}, J$ 7.3 Hz), 7.55-7.58 (4H, m), 7.64-7.66 $(2 \mathrm{H}, \mathrm{dt}, J 1.0,7.3 \mathrm{~Hz}), 7.80(1 \mathrm{H}, \mathrm{t}, J 1.0 \mathrm{~Hz}) . \delta_{\mathrm{C}}\left(\mathrm{CDCl}_{3}, 100.6 \mathrm{MHz}\right): 21.2,125.9,126.0(2 \mathrm{C})$, $127.1(2 \mathrm{C}), 127.3$ (2C), 127.4, 128.8(2C), 129.2, $129.6(2 \mathrm{C}), 137.2,138.3,141.3,141.7,141.8 . \mathrm{m} / \mathrm{z}$ (EI) $244\left(\mathrm{M}^{+}, 100 \%\right), 228(13), 215(5), 202(6), 165$ (13).

2”-Methoxy-[1,1';3',1”]terphenyl, 4j. Pale yellow oil. Purified by column chromatography, $\mathrm{R}_{\mathrm{f}}$ 0.3 (hexane- $\mathrm{CH}_{2} \mathrm{Cl}_{2}, 4: 1$ ). $v_{\max }$ (thin film) $/ \mathrm{cm}^{-1} 3058(\mathrm{CH}), 3028(\mathrm{CH}), 3001(\mathrm{CH}), 1598(\mathrm{CC})$, 1495 (CC), 1473 (CC), 1463 (CC), 1449 (CC), 1435 (CC), 1405 (CC), 897 (CH), 822 (CH), 755 (CH), $741(\mathrm{CH}), 703(\mathrm{CH}) . \delta_{\mathrm{H}}\left(\mathrm{CDCl}_{3}, 400 \mathrm{MHz}\right): 3.85(3 \mathrm{H}, \mathrm{s}), 7.02(1 \mathrm{H}, \mathrm{d}, J 8.3 \mathrm{~Hz}), 7.06(1 \mathrm{H}, \mathrm{td}$, $J$ 1.0, 7.5 Hz), 7.34-7.40 (3H, m), $7.46(2 \mathrm{H}, \mathrm{t}, J 7.1 \mathrm{~Hz}), 7.50(1 \mathrm{H}, \mathrm{d}, J 7.5 \mathrm{~Hz}), 7.54(1 \mathrm{H}, \mathrm{dt}, J 1.0$, $7.5 \mathrm{~Hz}), 7.57(1 \mathrm{H}, \mathrm{dt}, J 1.0,7.5 \mathrm{~Hz}), 7.63-7.66(2 \mathrm{H}, \mathrm{m}), 7.77(1 \mathrm{H}, \mathrm{t}, J 1.0 \mathrm{~Hz}) . \delta_{\mathrm{C}}\left(\mathrm{CDCl}_{3}, 100.6\right.$ MHz): 55.7, 111.3, 121.0, 125.9, 127.3, 127.4 (2C), 128.5, 128.6, 128.7, 128.8 (2C), 128.9, 130.7, 131.0, 139.1, 141.1, 141.5, 156.6. m/z (EI) $260\left(\mathrm{M}^{+}, 100 \%\right), 245$ (14), 226 (20), 215 (24), 202 (18), $142(18)$

2”-Methoxy-4-methyl-[1,1';3’,1”]terphenyl, 4k. Yellow solid. Purified by column chromatography, $R_{f} 0.3$ (hexane- $\mathrm{CH}_{2} \mathrm{Cl}_{2}, 4: 1$ ). mp 76-77 ${ }^{\circ} \mathrm{C}$. Found: C, 87.5; H, 6.65. $\mathrm{C}_{20} \mathrm{H}_{18} \mathrm{O}$ requires $\mathrm{C}, 87.55 ; \mathrm{H}, 6.6 \%$. $\mathrm{v}_{\max }(\mathrm{KBr} \mathrm{disc}) / \mathrm{cm}^{-1} 3048(\mathrm{CH}), 3023(\mathrm{CH}), 3006(\mathrm{CH}), 1602(\mathrm{CC})$, 1495 (CC), 1472 (CC), 1460 (CC), 1460 (CC), 1434 (CC), 1419 (CC), 819 (CH), 794 (CH), 755 (CH), $741(\mathrm{CH}), 717(\mathrm{CH}, \mathrm{w}), 704(\mathrm{CH}), 684(\mathrm{CH}) . \delta_{\mathrm{H}}\left(\mathrm{CDCl}_{3}, 400 \mathrm{MHz}\right): 2.41(3 \mathrm{H}, \mathrm{s}), 7.02(1 \mathrm{H}$, d, J 8.3 Hz), $7.06(1 \mathrm{H}, \mathrm{td}, J$ 1.0, $7.5 \mathrm{~Hz}), 7.27(2 \mathrm{H}, \mathrm{d}, J 8.3 \mathrm{~Hz}), 7.33-7.40(2 \mathrm{H}, \mathrm{m}), 7.46-7.57(5 \mathrm{H}$, m), $7.75(1 \mathrm{H}, \mathrm{t}, J 1.0 \mathrm{~Hz}) . \delta_{\mathrm{C}}\left(\mathrm{CDCl}_{3}, 100.6 \mathrm{MHz}\right): 21.2,55.6,111.3,120.9,125.7,127.2(2 \mathrm{C})$, $128.3(2 \mathrm{C}), 128.4,128.8,129.5$ (2C), 130.7, 131.0, 137.0, 138.6, 139.0, 141.0, 156.6. m/z (EI) 274 $\left(\mathrm{M}^{+}, 100 \%\right), 259(17), 244(18), 239(6), 226(8), 215(20), 156(14)$.

4-Fluoro-[1,1';2',1']terphenyl, 5a. Purified by column chromatography, $R_{f} 0.5$ (hexane-EtOAc, 9:1), mp 76-77 ${ }^{\circ} \mathrm{C}$ (lit. ${ }^{16} 77.5-79{ }^{\circ} \mathrm{C}$, hexane). Found: C, 87.2; H, 5.3. $\mathrm{C}_{18} \mathrm{H}_{13} \mathrm{~F}$ requires $\mathrm{C}, 87.07$; , 
5.28\%. $v_{\max }\left(\mathrm{KBr}\right.$ disc) $/ \mathrm{cm}^{-1} 3060(\mathrm{CH}), 3023(\mathrm{CH}), 1602$ (CC), 1498 (CC), 1474 (CC), 1449 (CC), $1433(\mathrm{CC}), 1402(\mathrm{CC}), 883(\mathrm{CH}), 842(\mathrm{CH}), 821(\mathrm{CH}), 779(\mathrm{CH}), 745(\mathrm{CH}), 703(\mathrm{CH}) . \delta_{\mathrm{H}}\left(\mathrm{CDCl}_{3}\right.$, $400 \mathrm{MHz}): 6.97$ (2H, t, J 8.8 Hz), $7.16(2 \mathrm{H}, \mathrm{dd}, J$ 5.4, $8.7 \mathrm{~Hz}), 7.19-7.21(2 \mathrm{H}, \mathrm{m}), 7.26-7.32(3 \mathrm{H}$, m), 7.47-7.49 (4H, m). $\delta_{\mathrm{C}}\left(\mathrm{CDCl}_{3}, 100.6 \mathrm{MHz}\right): 114.9(2 \mathrm{C}, \mathrm{d}, J 21.33 \mathrm{~Hz}), 126.64,127.66,127.9$, 128.0 (2C), 129.9 (2C), 130.6, 130.7, 131.4 (2C, d, J 7.87), 137.5, 139.6, 140.7, 141.4, 161.8 (d, J 245.74). m/z (EI) $248\left(\mathrm{M}^{+}, 100 \%\right), 233$ (31), 227 (24), 215 (6).

4-Methoxy-[1,1';2',1']terphenyl, 5b. Pale yellow oil. Purified by column chromatography, $R_{f}$ 0.6 (hexane-EtOAc, 9:1), low-melting solid (lit. ${ }^{17} 61.5-62.5{ }^{\circ} \mathrm{C}$ ). Found: C, 87.7; H, 6.2. $\mathrm{C}_{19} \mathrm{H}_{16} \mathrm{O}$ requires C, 87.66; H, 6.19\%. $v_{\max }\left(\right.$ thin film)/ $\mathrm{cm}^{-1} 3057(\mathrm{CH}), 3022(\mathrm{CH}), 3001(\mathrm{CH}), 2835$ (MeOAr), 1610 (CC), 1498 (CC), 1475 (CC), 1450 (CC), 1434 (CC), 1410 (CC), 1247 (MeOC), $1178(\mathrm{CO}), 1039(\mathrm{MeOC}), 875(\mathrm{CH}), 833(\mathrm{CH}), 806(\mathrm{CH}), 777(\mathrm{CH}), 745(\mathrm{CH}), 701(\mathrm{CH}) . \delta_{\mathrm{H}}$ $\left(\mathrm{CDCl}_{3}, 400 \mathrm{MHz}\right): 3.84(3 \mathrm{H}, \mathrm{s}), 6.85(2 \mathrm{H}, \mathrm{d}, J 8.7 \mathrm{~Hz}), 7.16(2 \mathrm{H}, \mathrm{d}, J$ 8.7 Hz), 7.24-7.34 (5H, m), 7.47-7.54 (4H, m). $\delta_{\mathrm{C}}\left(\mathrm{CDCl}_{3}, 100.6 \mathrm{MHz}\right): 55.2,113.4(2 \mathrm{C}), 126.5,127.2,127.6,128.0(2 \mathrm{C})$, 130.0 (2C), 130.65, 130.75, 131.0 (2C), 134.0, 140.2, 140.55, 141.8, 158.4. m/z (EI) $260\left(\mathrm{M}^{+}\right.$, 100\%), $229(29), 215(33), 202(27), 165(5)$.

1-[1,1';2',1']terphenyl-4-yl-ethanone, 5c. White solid. Purified by column chromatography, $R_{f}$ 0.25 (hexane-EtOAc, 5.6:1), mp 94-95 ${ }^{\circ} \mathrm{C}$ (lit. ${ }^{18}$ 93.5-94 ${ }^{\circ} \mathrm{C}$ ). Found: $\mathrm{C}, 88.2 ; \mathrm{H}, 5.9 . \mathrm{C}_{20} \mathrm{H}_{16} \mathrm{O}$ requires C, 88.20; H, 5.92\%. $\cup_{\max }(\mathrm{KBr}$ disc $) / \mathrm{cm}^{-1} 3062(\mathrm{CH}), 3024(\mathrm{CH}), 2999(\mathrm{CH}), 1610(\mathrm{CC})$, 1498 (CC), 1474 (CC), 1451 (CC), 1432 (CC), 1402 (CC), 875 (CH), 835 (CH), 780 (CH), 742 $(\mathrm{CH}), 701(\mathrm{CH}) . \delta_{\mathrm{H}}\left(\mathrm{CDCl}_{3}, 400 \mathrm{MHz}\right): 2.61(3 \mathrm{H}, \mathrm{s}), 7.17-7.19(2 \mathrm{H}, \mathrm{m}), 7.25-7.27(3 \mathrm{H}, \mathrm{m}), 7.29$ (2H, d, $J$ 8.4 Hz), 7.47-7.51 (4H, m), $7.86(2 \mathrm{H}, \mathrm{d}, J 8.4 \mathrm{~Hz}) . \delta_{\mathrm{C}}\left(\mathrm{CDCl}_{3}, 100.6 \mathrm{MHz}\right): 26.7,126.8$, 127.7, 128.1 (2C), 128.15 (2C), 128.3, 129.9 (2C), 130.15 (2C), 130.5, 130.9, 135.1, 139.4, 140.7, 141.1, 146.7, 197.9. m/z (EI) $272\left(\mathrm{M}^{+}, 68 \%\right), 257$ (100), 228 (48), 202 (15), 113 (12). 
2-Methoxy-[1,1';2',1"]terphenyl, 5d. ${ }^{19}$ Thick oil. Purified by column chromatography, $R_{f} 0.4$ (hexane-EtOAc, 19:1). Found: C, 87.6; H, 6.1. $\mathrm{C}_{19} \mathrm{H}_{16} \mathrm{O}$ requires $\mathrm{C}, 87.66 ; \mathrm{H}, 6.19 \%$. $\mathrm{v}_{\max }$ (thin film)/cm ${ }^{-1} 3057$ (CH), $3021(\mathrm{CH}), 3003(\mathrm{CH}), 2835$ (MeOAr), 1600 (CC), 1494 (CC), 1475 (CC), 1462 (CC), 1449 (CC), 1432 (CC), 1398 (CC), 1248 (MeOC), 1181 (CO), 1029 (MeOC), 874 (CH), $848(\mathrm{CH}), 804(\mathrm{CH}), 766(\mathrm{CH}), 742(\mathrm{CH}), 701(\mathrm{CH}) . \delta_{\mathrm{H}}\left(\mathrm{CDCl}_{3}, 400 \mathrm{MHz}\right): 3.47(3 \mathrm{H}, \mathrm{s}), 6.83(1 \mathrm{H}$, d, J $8.3 \mathrm{~Hz}), 7.05(1 \mathrm{H}, \mathrm{td}, J$ 1.0, $7.4 \mathrm{~Hz}), 7.25-7.37(7 \mathrm{H}, \mathrm{m}), 7.53-7.60(4 \mathrm{H}, \mathrm{m}) . \delta_{\mathrm{C}}\left(\mathrm{CDCl}_{3}, 100.6\right.$ $\mathrm{MHz}): 55.0,110.85,120.55,126.4,127.3,127.6$ (2C), 127.7, 128.7, 129.1 (2C), 129.8, 130.9, 131.1, 131.8, 137.4, 141.8, 142.3, 156.4. m/z (EI) $260\left(\mathrm{M}^{+}, 100 \%\right), 229$ (58), 215 (24), 202 (16), $165(5), 113(10)$.

1-(4-Methoxy-[1,1';2',1']terphenyl-4"'-yl)-ethanone, 5e. White solid. Purified by column chromatography. $R_{f} 0.2\left(\mathrm{CH}_{2} \mathrm{Cl}_{2}\right)$, mp $148-149{ }^{\circ} \mathrm{C}$. Found: C, 83.4; H, 6.0. $\mathrm{C}_{21} \mathrm{H}_{18} \mathrm{O}_{2}$ requires $\mathrm{C}$, 83.42; H, 6.00\%. v $v_{\max }\left(\mathrm{KBr}\right.$ disc) $/ \mathrm{cm}^{-1} 3066(\mathrm{CH}), 3035(\mathrm{CH}), 2844$ (MeOAr), 1683 (CO, vs), 1605 (CC), 1476 (CC), 1456 (CC), 1441 (CC), 1243 (MeOC), 1179 (CO), 1032 (MeOC), 834 (CH), 819 (CH), $776(\mathrm{CH}), 738(\mathrm{CH}), 725(\mathrm{CH}), 688(\mathrm{CH}) . \delta_{\mathrm{H}}\left(\mathrm{CDCl}_{3}, 400 \mathrm{MHz}\right): 2.61(3 \mathrm{H}, \mathrm{s}), 3.81(3 \mathrm{H}, \mathrm{s})$, $6.79(2 \mathrm{H}, \mathrm{d}, J$ 8.7 Hz), 7.07 (2H, d, J 8.7 Hz), 7.28 (2H, d, J 8.7 Hz), 7.43-7.49 (4H, m), 7.85 (2H, d, $J \quad 8.4 \mathrm{~Hz}) . \delta_{\mathrm{C}}\left(\mathrm{CDCl}_{3}, 100.6 \mathrm{MHz}\right): 26.7,55.2,113.6(2 \mathrm{C}), 127.35,128.1$ (2C), 128.3, 130.1 (2C), 130.5, 130.8, 131.0 (2C), 133.4, 135.1, 139.3, 140.3, 146.9, 158.6, 197.9. m/z (EI) $302\left(\mathrm{M}^{+}\right.$, 100\%), 287 (65), 259 (9), $226(22), 215$ (49), 189 (15).

1-(4-Fluoro-[1,1';2',1']terphenyl-4''-yl)-ethanone, 5f. White solid. Purified by column chromatography. $R_{f} 0.05\left(\mathrm{CH}_{2} \mathrm{Cl}_{2}\right)$, mp $109-110^{\circ} \mathrm{C}$. Found: $\mathrm{C}, 82.7$; H, 5.2. $\mathrm{C}_{20} \mathrm{H}_{15} \mathrm{FO}$ requires $\mathrm{C}$, 82.74; H, 5.21\%. v $\max (\mathrm{KBr} \operatorname{disc}) / \mathrm{cm}^{-1} 3071(\mathrm{CH}, \mathrm{w}), 3059(\mathrm{CH}, \mathrm{w}), 1682(\mathrm{CO}, \mathrm{s}), 1603(\mathrm{CC}, \mathrm{s})$, 1478 (CC, s), 1432 (CC, w), 1425 (CC, w), 1403 (CC), 835 (CH), $828(\mathrm{CH}), 817$ (CH), 767 (CH, s). $\delta_{\mathrm{H}}\left(\mathrm{CDCl}_{3}, 400 \mathrm{MHz}\right): 2.61(3 \mathrm{H}, \mathrm{s}), 6.95(2 \mathrm{H}, \mathrm{t}, J 8.7 \mathrm{~Hz}), 7.11(2 \mathrm{H}, \mathrm{dd}, J$ 5.2, $8.7 \mathrm{~Hz}), 7.26$ $(2 \mathrm{H}, \mathrm{d}, J 8.4 \mathrm{~Hz}), 7.44-7.51(4 \mathrm{H}, \mathrm{m}), 7.87\left(2 \mathrm{H}, \mathrm{d}, J\right.$ 8.4 Hz). $\delta_{\mathrm{C}}\left(\mathrm{CDCl}_{3}, 100.6 \mathrm{MHz}\right): 26.7,115.1$ (2C, d, J $21 \mathrm{~Hz}), 127.8,128.16$ (2C), 128.32, 130.1 (2C), 130.5, 130.7, 131.4 (2C, d, J 7.9 Hz), 
135.2, 137.0, 139.4, 139.6, 146.4, 161.9 (d, J 246 Hz), 197.9. m/z (EI) 290 (M+, 57\%), 275 (100), 246 (30), 226 (26), 113 (11).

1-(2-Methoxy-[1,1';2',1']terphenyl-4'-yl)-ethanone, 5g. White solid. Purified by column chromatography. $R_{f} 0.2\left(\mathrm{CH}_{2} \mathrm{Cl}_{2}\right)$, mp $90-91^{\circ} \mathrm{C}$. Found: C, 83.3; $\mathrm{H}, 6.0 . \mathrm{C}_{21} \mathrm{H}_{18} \mathrm{O}_{2}$ requires $\mathrm{C}, 83.42$; H, 6.00\%. U $v_{\max }\left(\mathrm{KBr}\right.$ disc) $/ \mathrm{cm}^{-1} 3058(\mathrm{CH}), 3018(\mathrm{CH}), 2837$ (MeOAr), 1607 (CC), 1496 (CC), 1476 (CC), 1449 (CC), 1430 (CC), 1407 (CC), 1254 (MeOC), 1185 (CO), 1051 (MeOC), 879 (CH), $832(\mathrm{CH}), 804(\mathrm{CH}), 765(\mathrm{CH}), 748(\mathrm{CH}), 704(\mathrm{CH}) . \delta_{\mathrm{H}}\left(\mathrm{CDCl}_{3}, 400 \mathrm{MHz}\right): 2.60(3 \mathrm{H}, \mathrm{s}), 3.38(3 \mathrm{H}$, s), $6.74(1 \mathrm{H}, \mathrm{d}, J 8.3 \mathrm{~Hz}), 6.99(1 \mathrm{H}, \mathrm{td}, J$ 1.0, $7.4 \mathrm{~Hz}), 7.24(1 \mathrm{H}, \mathrm{dd}, J$ 1.7, $8.4 \mathrm{~Hz}), 7.27-7.31(3 \mathrm{H}$, m), 7.44-7.51 (4H, m), $7.84(2 \mathrm{H}, \mathrm{d}, J \quad 8.5 \mathrm{~Hz}) . \delta_{\mathrm{C}}\left(\mathrm{CDCl}_{3}, 100.6 \mathrm{MHz}\right): 26.7,54.9,110.8,120.7$, 127.8 (2C), 128.0, 129.0, 129.1 (2C), 129.6, 130.2, 131.2, 131.5, 135.0, 137.4, 140.6, 147.4, 156.1, 198.0. m/z (EI) $302\left(\mathrm{M}^{+}, 84 \%\right), 287$ (100), 244 (11), 226 (32), 215 (34), 189 (12).

4'-Fluoro-2-methoxy-[1,1';2',1']terphenyl, 5h. Thick oil. Purified by column chromatography. $R_{f} 0.15$ (hexane-EtOAc, 49:1). Found: C, 82.0; H, 5.4. $\mathrm{C}_{19} \mathrm{H}_{15} \mathrm{FO}$ requires $\mathrm{C}, 81.99 ; \mathrm{H}, 5.43 \%$. $\mathrm{v}_{\max }$ (thin film)/ $\mathrm{cm}^{-1} 3058(\mathrm{CH}), 3022(\mathrm{CH}), 2999(\mathrm{CH}), 2834$ (MeOAr), 1602 (CC), $1496(\mathrm{CC}), 1476$ (CC), 1452 (CC), 1432 (CC), 1402 (CC), 1246 (MeOC), 1180 (CO), 1049 (MeOC), 876 (CH), 837 (CH), $801(\mathrm{CH}), 762(\mathrm{CH}), 735(\mathrm{CH}), 704(\mathrm{CH}) . \delta_{\mathrm{H}}\left(\mathrm{CDCl}_{3}, 400 \mathrm{MHz}\right): 3.46(3 \mathrm{H}, \mathrm{s}), 6.79(1 \mathrm{H}, \mathrm{d}, J$ $8.8 \mathrm{~Hz}), 6.95(2 \mathrm{H}, \mathrm{t}, J 8.8 \mathrm{~Hz}), 7.02(1 \mathrm{H}, \mathrm{td}, J 1.0,7.4 \mathrm{~Hz}) ; 7.18(2 \mathrm{H}, \mathrm{dd}, J 5.5,8.8 \mathrm{~Hz}), 7.25(1 \mathrm{H}$, dd, $J 1.7,7.4 \mathrm{~Hz}), 7.32(1 \mathrm{H}, \mathrm{td}, J 1.8,7.4 \mathrm{~Hz}), 7.45-7.52(4 \mathrm{H}, \mathrm{m}) . \delta_{\mathrm{C}}\left(\mathrm{CDCl}_{3}, 100.6 \mathrm{MHz}\right): 55.0$, 110.8, 114.4 (2C, d, J $21 \mathrm{~Hz}), 120.6,127.4,127.7,128.8,129.6,130.5$ (2C, d, J 7.7 Hz), 130.6, 131.0, 131.6, 137.4, 138.3, 140.8, 156.2, 161.66 (d, J $245 \mathrm{~Hz}) . m / z(\mathrm{EI}) 278\left(\mathrm{M}^{+}, 100 \%\right), 247$ (24), $233(28), 220(16), 183(8)$.

4-Fluoro-4'-methoxy-[1,1';2',1']terphenyl, 5i. Thick oil. Purified by column chromatography. $R_{f} 0.15$ (hexane-EtOAc, 49:1). Found: C, 82.0; H, 5.3. $\mathrm{C}_{19} \mathrm{H}_{15} \mathrm{FO}$ requires $\mathrm{C}, 81.99 ; \mathrm{H}, 5.43 \%$. $\mathrm{v}_{\max }$ (thin film) $/ \mathrm{cm}^{-1} 3058(\mathrm{CH}), 3037(\mathrm{CH}), 3001(\mathrm{CH}), 2836(\mathrm{MeOAr}), 1607$ (CC), 1508 (CC), 1476 (CC), 1452 (CC), 1409 (CC), 1246 (MeOC), 1178 (CO), 1038 (MeOC), 877 (CH), 839 (CH), 804 
(CH), $762(\mathrm{CH}), 734(\mathrm{CH}), 702(\mathrm{CH}) . \delta_{\mathrm{H}}\left(\mathrm{CDCl}_{3}, 400 \mathrm{MHz}\right): 3.84(3 \mathrm{H}, \mathrm{s}), 6.83(2 \mathrm{H}, \mathrm{d}, \mathrm{J} 8.8 \mathrm{~Hz})$, $6.96(2 \mathrm{H}, \mathrm{t}, J 8.8 \mathrm{~Hz}), 7.10(2 \mathrm{H}, \mathrm{d}, J 8.8 \mathrm{~Hz}), 7.17(2 \mathrm{H}, \mathrm{dd}, J 5.4,8.8 \mathrm{~Hz}) ; 7.42-7.47(4 \mathrm{H}, \mathrm{m}) . \delta_{\mathrm{C}}$ ( $\left.\mathrm{CDCl}_{3}, 100.6 \mathrm{MHz}\right): 55.2,113.5$ (2C), 114.9 (2C, d, J $\left.21 \mathrm{~Hz}\right), 127.3,127.7,130.6,130,7,131.0$ (2C), 131.4 (2C, d, J 7.9 Hz), 133.7, 137.7, 139.5, 140.2, 158.4, 161.7 (d, J $246 \mathrm{~Hz}) . \mathrm{m} / \mathrm{z}$ (EI) 278 $\left(\mathrm{M}^{+}, 100 \%\right), 245$ (12), 234 (14), 220 (31), 215 (20), 207 (11), 183 (9).

2,4'-Dimethoxy-[1,1';2',1']terphenyl, 5j. Thick oil. Purified by column chromatography. $R_{f}$ 0.25 (hexane-EtOAc, 19:1). Found: C, 82.8; H, 6.3. $\mathrm{C}_{20} \mathrm{H}_{18} \mathrm{O}_{2}$ requires C, 82.73; H, 6.25\%. $\mathrm{v}_{\max }$ (thin film) $/ \mathrm{cm}^{-1} 3058(\mathrm{CH}), 3021(\mathrm{CH}), 3000(\mathrm{CH}), 2834$ (MeOAr), $1610(\mathrm{CC}), 1497(\mathrm{CC}), 1476$ (CC), 1462 (CC), 1432 (CC), 1410 (CC), 1246 (MeOC), 1179 (CO), 1034 (MeOC), 875 (CH), 834 (CH), $809(\mathrm{CH}), 761(\mathrm{CH}), 735(\mathrm{CH}), 703(\mathrm{CH}) . \delta_{\mathrm{H}}\left(\mathrm{CDCl}_{3}, 400 \mathrm{MHz}\right): 3.44(3 \mathrm{H}, \mathrm{s}), 3.79(3 \mathrm{H}, \mathrm{s})$, $6.76(2 \mathrm{H}, \mathrm{d}, J 8.8 \mathrm{~Hz}), 6.76-6.78(1 \mathrm{H}, \mathrm{m}), 6.96(1 \mathrm{H}, \mathrm{td}, J 1.0,7.4 \mathrm{~Hz}), 7.10(2 \mathrm{H}, \mathrm{d}, J 8.8 \mathrm{~Hz}), 7.20$ (1H, dd, J 1.7, 7.4 Hz), 7.25-7.29 (1H, m), 7.40-7.46 (4H, m). $\delta_{\mathrm{C}}\left(\mathrm{CDCl}_{3}, 100.6 \mathrm{MHz}\right): 55.1,55.2$, $110.8,113.0$ (2C), 120.4, 126.8, 127.6, 128.5, 129.7, 130.0 (2C), 130.9, 131.0, 131.6, 134.75, 137.2, 141.3, 156.3, 158.1. m/z (EI) $290\left(\mathrm{M}^{+}, 100 \%\right), 259$ (15), 215 (28), $202(21), 189$ (9), 152 (6). 


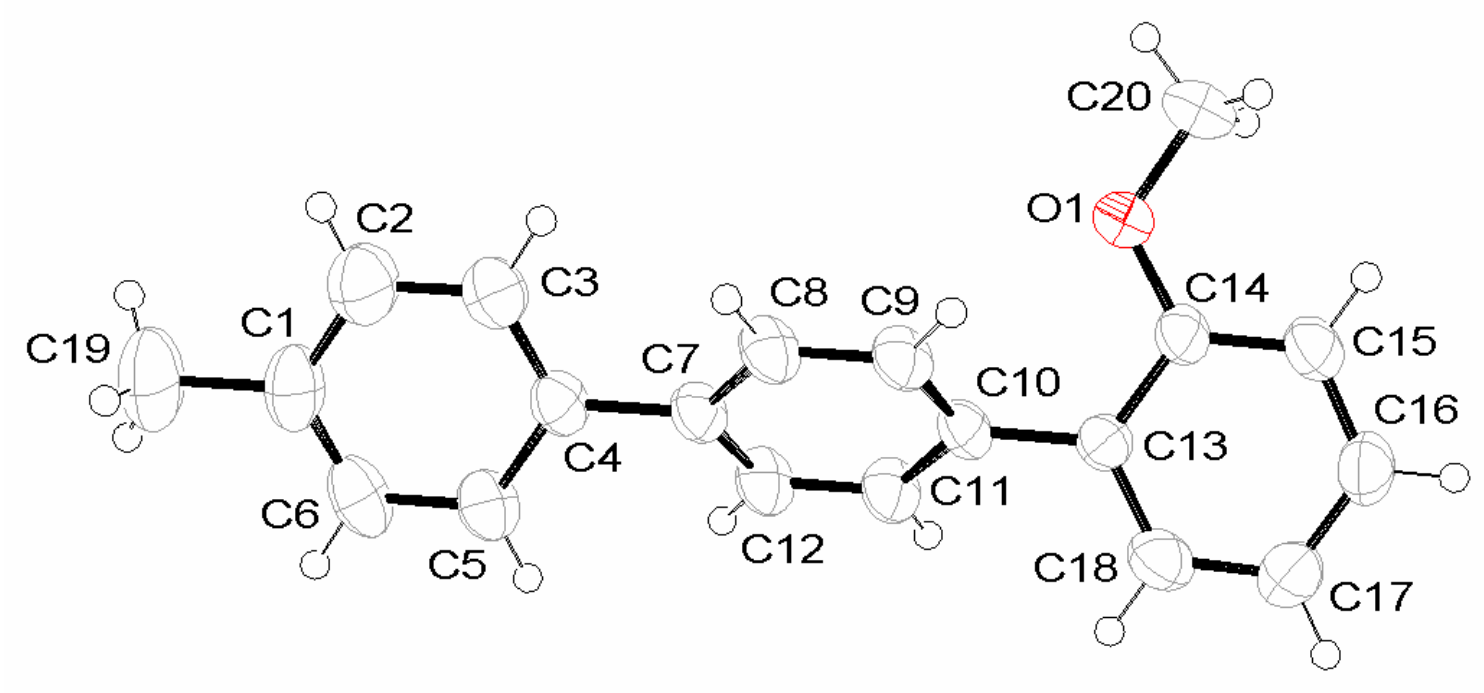

Figure S1. ORTEP drawing of the molecular structure of compound 3k.

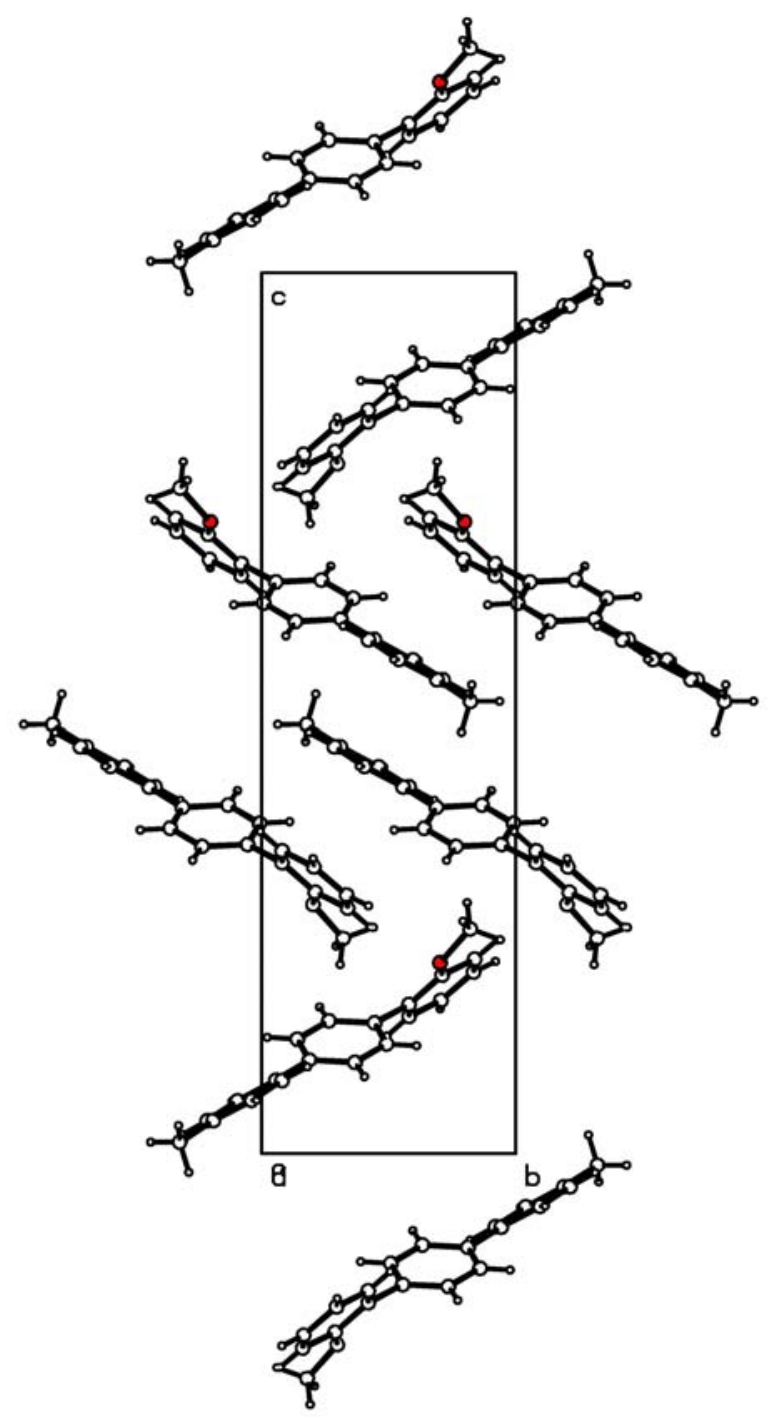

Figure S2. Crystal packing of 3k. 
Table S1. Crystal data and structure refinement for compound 3k.

Identification code

Empirical formula

Formula weight

Temperature

Wavelength

Crystal system

Space group

Unit cell dimensions

Volume

Z

Density (calculated)

Absorption coefficient

$\mathrm{F}(000)$

Crystal size

Theta range for data collection

Index ranges

Reflections collected

Independent reflections

Completeness to theta $=23.96^{\circ}$

Absorption correction

Max. and min. transmission

Refinement method

Data / restraints / parameters

Goodness-of-fit on $\mathrm{F}^{2}$

Final $\mathrm{R}$ indices $[\mathrm{I}>2 \operatorname{sigma}(\mathrm{I})]$

$\mathrm{R}$ indices (all data)

Extinction coefficient

Largest diff. peak and hole vi38jiln

$\mathrm{C}_{20} \mathrm{H}_{18} \mathrm{O}$

274.34

293(2) K

$0.71069 \AA$

Monoclinic

P 21/c

$\mathrm{a}=7.601(5) \AA$

$\alpha=90.000(5)^{\circ}$.

$\mathrm{b}=7.577(5) \AA$

$\beta=94.107(5)^{\circ}$.

$\mathrm{c}=26.341(5) \AA$

$\gamma=90.000(5)^{\circ}$.

$1513.2(14) \AA^{3}$

4

$1.204 \mathrm{Mg} / \mathrm{m}^{3}$

$0.072 \mathrm{~mm}^{-1}$

584

$0.36 \times 0.28 \times 0.20 \mathrm{~mm}^{3}$

1.55 to $23.96^{\circ}$.

$-8<=\mathrm{h}<=8,-8<=\mathrm{k}<=8,-29<=\mathrm{l}<=29$

9325

$2358[\mathrm{R}(\mathrm{int})=0.0569]$

$99.1 \%$

Semi-empirical from equivalents

0.986 and 0.976

Full-matrix least-squares on $\mathrm{F}^{2}$

$2358 / 0 / 193$

1.130

$\mathrm{R} 1=0.0819, \mathrm{wR} 2=0.2257$

$\mathrm{R} 1=0.1175, \mathrm{wR} 2=0.2499$

$0.011(3)$

0.294 and -0.233 e. $\AA^{-3}$

Table S2. Bond lengths $[\AA]$ and angles $\left[{ }^{\circ}\right]$ for compound $3 \mathbf{k}$.

\begin{tabular}{ll}
\hline $\mathrm{O}(1)-\mathrm{C}(14)$ & $1.367(5)$ \\
$\mathrm{O}(1)-\mathrm{C}(20)$ & $1.427(6)$ \\
$\mathrm{C}(14)-\mathrm{C}(15)$ & $1.389(6)$ \\
$\mathrm{C}(14)-\mathrm{C}(13)$ & $1.401(6)$ \\
$\mathrm{C}(10)-\mathrm{C}(9)$ & $1.383(6)$
\end{tabular}




\begin{tabular}{|c|c|}
\hline $\mathrm{C}(10)-\mathrm{C}(11)$ & $1.402(6)$ \\
\hline$C(10)-C(13)$ & $1.488(6)$ \\
\hline$C(13)-C(18)$ & $1.376(6)$ \\
\hline $\mathrm{C}(9)-\mathrm{C}(8)$ & $1.374(6)$ \\
\hline $\mathrm{C}(9)-\mathrm{H}(9)$ & 0.9300 \\
\hline$C(8)-C(7)$ & $1.400(6)$ \\
\hline $\mathrm{C}(8)-\mathrm{H}(8)$ & 0.9300 \\
\hline$C(4)-C(3)$ & $1.383(7)$ \\
\hline$C(4)-C(5)$ & $1.401(6)$ \\
\hline$C(4)-C(7)$ & $1.479(6)$ \\
\hline $\mathrm{C}(18)-\mathrm{C}(17)$ & $1.378(7)$ \\
\hline $\mathrm{C}(18)-\mathrm{H}(18)$ & 0.9300 \\
\hline$C(6)-C(5)$ & $1.375(7)$ \\
\hline$C(6)-C(1)$ & $1.378(8)$ \\
\hline $\mathrm{C}(6)-\mathrm{H}(6)$ & 0.9300 \\
\hline$C(15)-C(16)$ & $1.376(7)$ \\
\hline $\mathrm{C}(15)-\mathrm{H}(15)$ & 0.9300 \\
\hline $\mathrm{C}(11)-\mathrm{C}(12)$ & $1.380(6)$ \\
\hline $\mathrm{C}(11)-\mathrm{H}(11)$ & 0.9300 \\
\hline $\mathrm{C}(5)-\mathrm{H}(5)$ & 0.9300 \\
\hline $\mathrm{C}(16)-\mathrm{C}(17)$ & $1.373(7)$ \\
\hline $\mathrm{C}(16)-\mathrm{H}(16)$ & 0.9300 \\
\hline$C(7)-C(12)$ & $1.384(6)$ \\
\hline $\mathrm{C}(17)-\mathrm{H}(17)$ & 0.9300 \\
\hline $\mathrm{C}(12)-\mathrm{H}(12)$ & 0.9300 \\
\hline$C(3)-C(2)$ & $1.378(7)$ \\
\hline $\mathrm{C}(3)-\mathrm{H}(3)$ & 0.9300 \\
\hline$C(2)-C(1)$ & $1.383(8)$ \\
\hline $\mathrm{C}(2)-\mathrm{H}(2)$ & 0.9300 \\
\hline$C(1)-C(19)$ & $1.509(7)$ \\
\hline $\mathrm{C}(20)-\mathrm{H}(20 \mathrm{~A})$ & 0.9600 \\
\hline $\mathrm{C}(20)-\mathrm{H}(20 \mathrm{~B})$ & 0.9600 \\
\hline $\mathrm{C}(20)-\mathrm{H}(20 \mathrm{C})$ & 0.9600 \\
\hline C(19)-H(19A) & 0.9600 \\
\hline C(19)-H(19B) & 0.9600 \\
\hline C(19)-H(19C) & 0.9600 \\
\hline $\mathrm{C}(14)-\mathrm{O}(1)-\mathrm{C}(20)$ & $118.0(4)$ \\
\hline $\mathrm{O}(1)-\mathrm{C}(14)-\mathrm{C}(15)$ & $123.4(4)$ \\
\hline $\mathrm{O}(1)-\mathrm{C}(14)-\mathrm{C}(13)$ & $116.6(4)$ \\
\hline
\end{tabular}




$\begin{array}{ll}\mathrm{C}(15)-\mathrm{C}(14)-\mathrm{C}(13) & 120.0(4) \\ \mathrm{C}(9)-\mathrm{C}(10)-\mathrm{C}(11) & 117.1(4) \\ \mathrm{C}(9)-\mathrm{C}(10)-\mathrm{C}(13) & 121.6(4) \\ \mathrm{C}(11)-\mathrm{C}(10)-\mathrm{C}(13) & 121.3(4) \\ \mathrm{C}(18)-\mathrm{C}(13)-\mathrm{C}(14) & 118.3(4) \\ \mathrm{C}(18)-\mathrm{C}(13)-\mathrm{C}(10) & 120.7(4) \\ \mathrm{C}(14)-\mathrm{C}(13)-\mathrm{C}(10) & 120.9(4) \\ \mathrm{C}(8)-\mathrm{C}(9)-\mathrm{C}(10) & 122.0(4) \\ \mathrm{C}(8)-\mathrm{C}(9)-\mathrm{H}(9) & 119.0 \\ \mathrm{C}(10)-\mathrm{C}(9)-\mathrm{H}(9) & 119.0 \\ \mathrm{C}(9)-\mathrm{C}(8)-\mathrm{C}(7) & 121.1(4) \\ \mathrm{C}(9)-\mathrm{C}(8)-\mathrm{H}(8) & 119.4 \\ \mathrm{C}(7)-\mathrm{C}(8)-\mathrm{H}(8) & 119.4 \\ \mathrm{C}(3)-\mathrm{C}(4)-\mathrm{C}(5) & 117.2(4) \\ \mathrm{C}(3)-\mathrm{C}(4)-\mathrm{C}(7) & 122.1(4) \\ \mathrm{C}(5)-\mathrm{C}(4)-\mathrm{C}(7) & 120.7(4) \\ \mathrm{C}(13)-\mathrm{C}(18)-\mathrm{C}(17) & 121.9(5) \\ \mathrm{C}(13)-\mathrm{C}(18)-\mathrm{H}(18) & 119.0 \\ \mathrm{C}(17)-\mathrm{C}(18)-\mathrm{H}(18) & 119.0 \\ \mathrm{C}(5)-\mathrm{C}(6)-\mathrm{C}(1) & 122.3(5) \\ \mathrm{C}(5)-\mathrm{C}(6)-\mathrm{H}(6) & 118.9 \\ \mathrm{C}(1)-\mathrm{C}(6)-\mathrm{H}(6) & 118.9 \\ \mathrm{C}(16)-\mathrm{C}(15)-\mathrm{C}(14) & 119.9(4) \\ \mathrm{C}(16)-\mathrm{C}(15)-\mathrm{H}(15) & 120.1 \\ \mathrm{C}(14)-\mathrm{C}(15)-\mathrm{H}(15) & 120.1 \\ \mathrm{C}(12)-\mathrm{C}(11)-\mathrm{C}(10) & 120.8(4) \\ \mathrm{C}(12)-\mathrm{C}(11)-\mathrm{H}(11) & 119.6 \\ \mathrm{C}(10)-\mathrm{C}(11)-\mathrm{H}(11) & 119.6 \\ \mathrm{C}(6)-\mathrm{C}(5)-\mathrm{C}(4) & 120.1(5) \\ \mathrm{C}(6)-\mathrm{C}(5)-\mathrm{H}(5) & 120.0 \\ \mathrm{C}(4)-\mathrm{C}(5)-\mathrm{H}(5) & 120.0 \\ \mathrm{C}(17)-\mathrm{C}(16)-\mathrm{C}(15) & 120.8(5) \\ \mathrm{C}(17)-\mathrm{C}(16)-\mathrm{H}(16) & 119.6 \\ \mathrm{C}(15)-\mathrm{C}(16)-\mathrm{H}(16) & 119.6 \\ \mathrm{C}(12)-\mathrm{C}(7)-\mathrm{C}(8) & 117.0(4) \\ \mathrm{C}(8)-\mathrm{C}(7)-\mathrm{C}(4) & 122.8(4) \\ \mathrm{C}(16)-\mathrm{C}(17)-\mathrm{C}(18) & 119.1(5) \\ \mathrm{C}(16)-\mathrm{C}(17)-\mathrm{H}(17) & 120.4 \\ \mathrm{C}(18)-\mathrm{C}(17)-\mathrm{H}(17) & 120.4 \\ & \end{array}$




\begin{tabular}{|c|c|}
\hline$C(11)-C(12)-C(7)$ & $122.0(4)$ \\
\hline $\mathrm{C}(11)-\mathrm{C}(12)-\mathrm{H}(12)$ & 119.0 \\
\hline $\mathrm{C}(7)-\mathrm{C}(12)-\mathrm{H}(12)$ & 119.0 \\
\hline$C(2)-C(3)-C(4)$ & $122.3(5)$ \\
\hline $\mathrm{C}(2)-\mathrm{C}(3)-\mathrm{H}(3)$ & 118.9 \\
\hline $\mathrm{C}(4)-\mathrm{C}(3)-\mathrm{H}(3)$ & 118.9 \\
\hline$C(3)-C(2)-C(1)$ & $120.2(6)$ \\
\hline $\mathrm{C}(3)-\mathrm{C}(2)-\mathrm{H}(2)$ & 119.9 \\
\hline $\mathrm{C}(1)-\mathrm{C}(2)-\mathrm{H}(2)$ & 119.9 \\
\hline$C(6)-C(1)-C(2)$ & $117.9(5)$ \\
\hline$C(6)-C(1)-C(19)$ & $121.5(6)$ \\
\hline$C(2)-C(1)-C(19)$ & $120.6(6)$ \\
\hline $\mathrm{O}(1)-\mathrm{C}(20)-\mathrm{H}(20 \mathrm{~A})$ & 109.5 \\
\hline $\mathrm{O}(1)-\mathrm{C}(20)-\mathrm{H}(20 \mathrm{~B})$ & 109.5 \\
\hline $\mathrm{H}(20 \mathrm{~A})-\mathrm{C}(20)-\mathrm{H}(20 \mathrm{~B})$ & 109.5 \\
\hline $\mathrm{O}(1)-\mathrm{C}(20)-\mathrm{H}(20 \mathrm{C})$ & 109.5 \\
\hline $\mathrm{H}(20 \mathrm{~A})-\mathrm{C}(20)-\mathrm{H}(20 \mathrm{C})$ & 109.5 \\
\hline $\mathrm{H}(20 \mathrm{~B})-\mathrm{C}(20)-\mathrm{H}(20 \mathrm{C})$ & 109.5 \\
\hline$C(1)-C(19)-H(19 A)$ & 109.5 \\
\hline $\mathrm{C}(1)-\mathrm{C}(19)-\mathrm{H}(19 \mathrm{~B})$ & 109.5 \\
\hline $\mathrm{H}(19 \mathrm{~A})-\mathrm{C}(19)-\mathrm{H}(19 \mathrm{~B})$ & 109.5 \\
\hline $\mathrm{C}(1)-\mathrm{C}(19)-\mathrm{H}(19 \mathrm{C})$ & 109.5 \\
\hline $\mathrm{H}(19 \mathrm{~A})-\mathrm{C}(19)-\mathrm{H}(19 \mathrm{C})$ & 109.5 \\
\hline H(19B)-C(19)-H(19C) & 109.5 \\
\hline
\end{tabular}

Symmetry transformations used to generate equivalent atoms: 
Table S3. UV/vis absorption maxima of terphenyl rings. ${ }^{a}$

\begin{tabular}{|c|c|c|c|c|}
\hline Compound & $\lambda_{\max } / \mathrm{nm}$ & $\varepsilon / \mathrm{dm}^{3} \mathrm{~mol}^{-1} \mathrm{~cm}^{-1}$ & $\lambda_{\max } / \mathrm{nm}$ & $\varepsilon / \mathrm{dm}^{3} \mathrm{~mol}^{-1} \mathrm{~cm}^{-1}$ \\
\hline $3 a$ & 204 & 42300 & 300 & 34000 \\
\hline $3 \mathbf{b}$ & 202 & 46400 & 302 & 36000 \\
\hline $3 c$ & 204 & 47200 & 306 & 36250 \\
\hline 3d & 206 & 49600 & 304 & 29300 \\
\hline $3 \mathbf{e}$ & 206 & 51600 & 312 & 38600 \\
\hline $3 f$ & 206 & 47000 & 278 & 30500 \\
\hline $3 g$ & 206 & 49100 & 282 & 34100 \\
\hline $3 \mathrm{~h}$ & 206 & 48600 & $268 \mathrm{sh}$ & 23500 \\
\hline $3 \mathbf{i}$ & 208 & 46700 & 282 & 32300 \\
\hline $3 \mathbf{j}$ & 206 & 51000 & $270 \mathrm{sh}$ & 24200 \\
\hline $3 \mathbf{k}$ & 208 & 49350 & $272 \mathrm{sh}$ & 24900 \\
\hline $4 a$ & 204 & 42200 & 264 & 31700 \\
\hline $4 \mathbf{b}$ & 204 & 40000 & 264 & 30300 \\
\hline $4 c$ & 206 & 41350 & $268 \mathrm{sh}$ & 35900 \\
\hline 4d & $212 \mathrm{sh}$ & 41450 & $284 \mathrm{sh}$ & 28750 \\
\hline $4 e$ & 208 & 41700 & 276 & 41000 \\
\hline $4 f$ & 206 & 40400 & 248 & 37600 \\
\hline $4 g$ & 206 & 41400 & 250 & 39150 \\
\hline $4 h$ & 208 & 43700 & $246 \mathrm{sh}$ & 31800 \\
\hline $4 \mathbf{i}$ & 208 & 43500 & 252 & 41500 \\
\hline $4 j$ & 208 & 50450 & $246 \mathrm{sh}$ & 36200 \\
\hline $4 \mathbf{k}$ & 210 & 44700 & $250 \mathrm{sh}$ & 32100 \\
\hline $5 a$ & 205 & 37600 & 233,249 & 22800,11600 \\
\hline $5 \mathbf{b}$ & 203 & 44400 & 242,256 & 22800,12400 \\
\hline $5 c$ & 203 & 40800 & 247,276 & 20800,14400 \\
\hline 5d & 204 & 39200 & 232,280 & 17600,2800 \\
\hline $5 e$ & 204 & 44400 & 256,274 & 26800,15600 \\
\hline
\end{tabular}




\begin{tabular}{|l|l|l|l|l|}
\hline $\mathbf{5 f}$ & 203 & 42400 & 247,276 & 21200,15600 \\
\hline $\mathbf{5 g}$ & 204 & 45600 & 248,277 & 17200,18400 \\
\hline $\mathbf{5 h}$ & 203 & 40400 & 231,273 & 19200,3200 \\
\hline $\mathbf{5 i}$ & 202 & 43600 & 241,259 & 22000,12000 \\
\hline $\mathbf{5 j}$ & 204 & 44000 & 241,264 & 14400,9600 \\
\hline
\end{tabular}

${ }^{a}$ Spectra was recorded of $2.5 \times 10^{-5} \mathrm{M}$ solutions in acetonitrile, sh $=$ shoulder. ${ }^{b}$ Both absorption bands display shoulders at lower wavelength. The more intense band was recorded. 
Figure S3. ${ }^{1} \mathrm{H}$ and ${ }^{13} \mathrm{C}$ NMR spectra of compound $\mathbf{3 a}$.
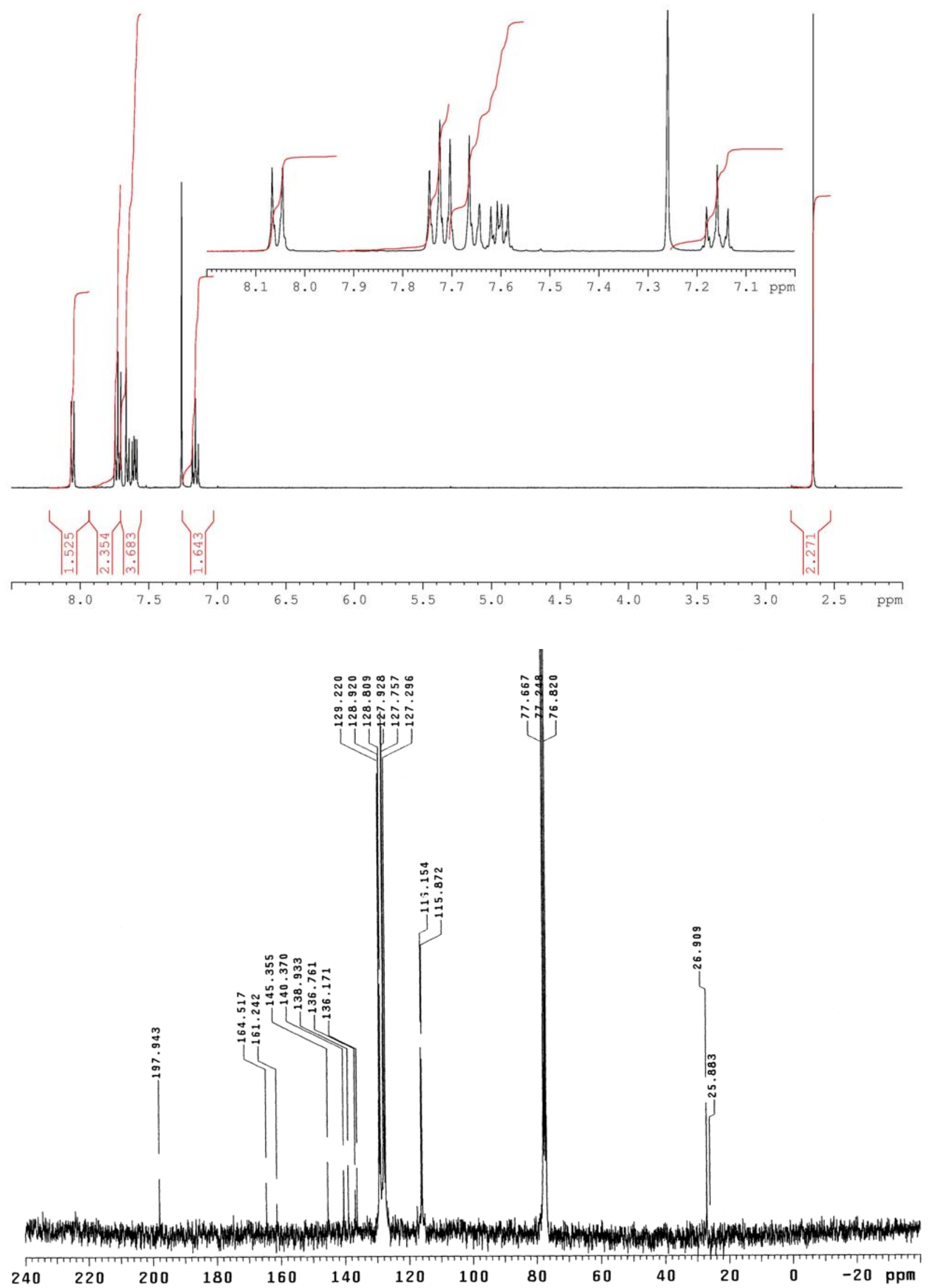
Figure S4. ${ }^{1} \mathrm{H}$ and ${ }^{13} \mathrm{C}$ NMR spectra of compound 3d.
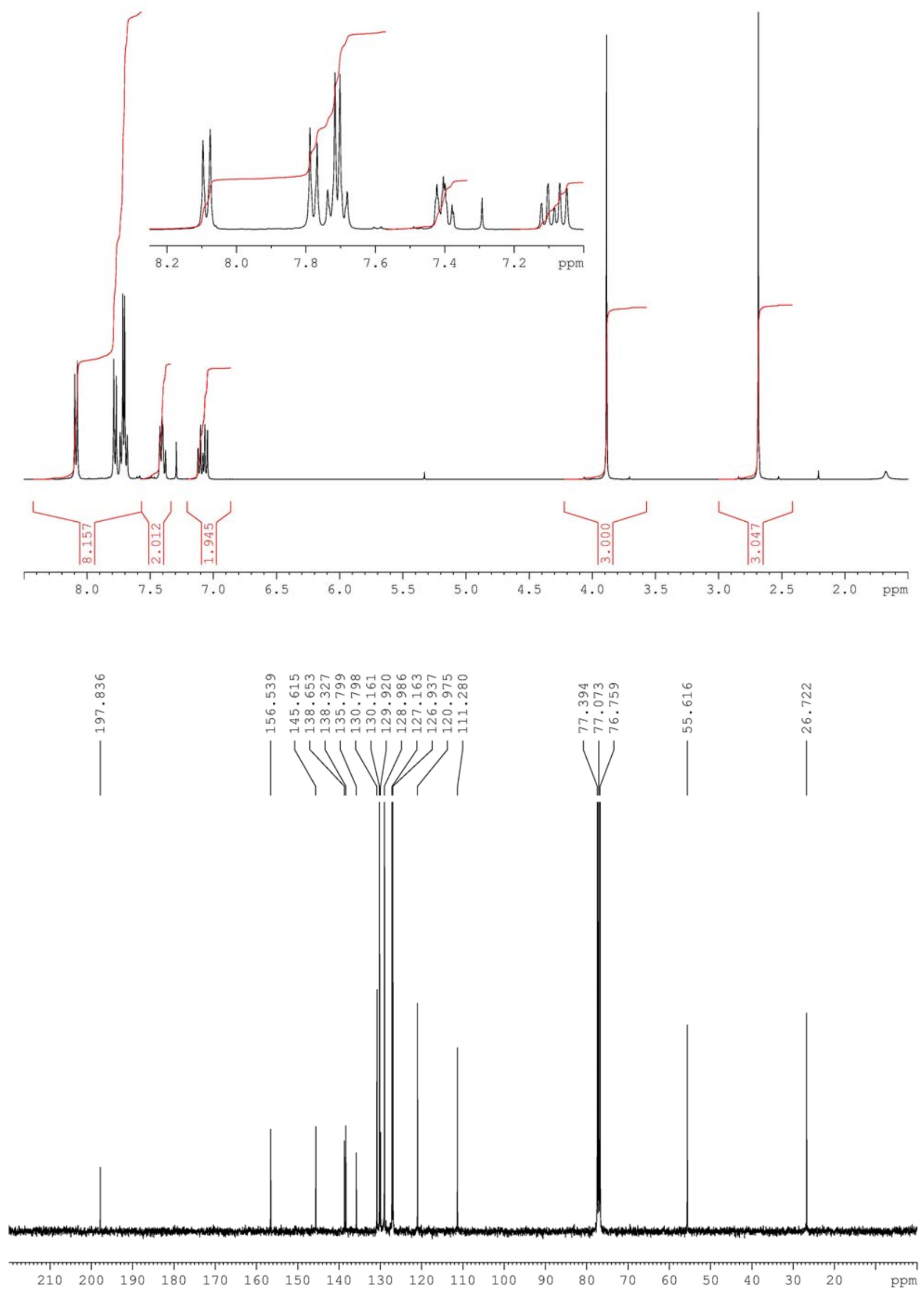
Figure S5. ${ }^{1} \mathrm{H}$ and ${ }^{13} \mathrm{C}$ NMR spectra of compound $3 \mathbf{e}$.
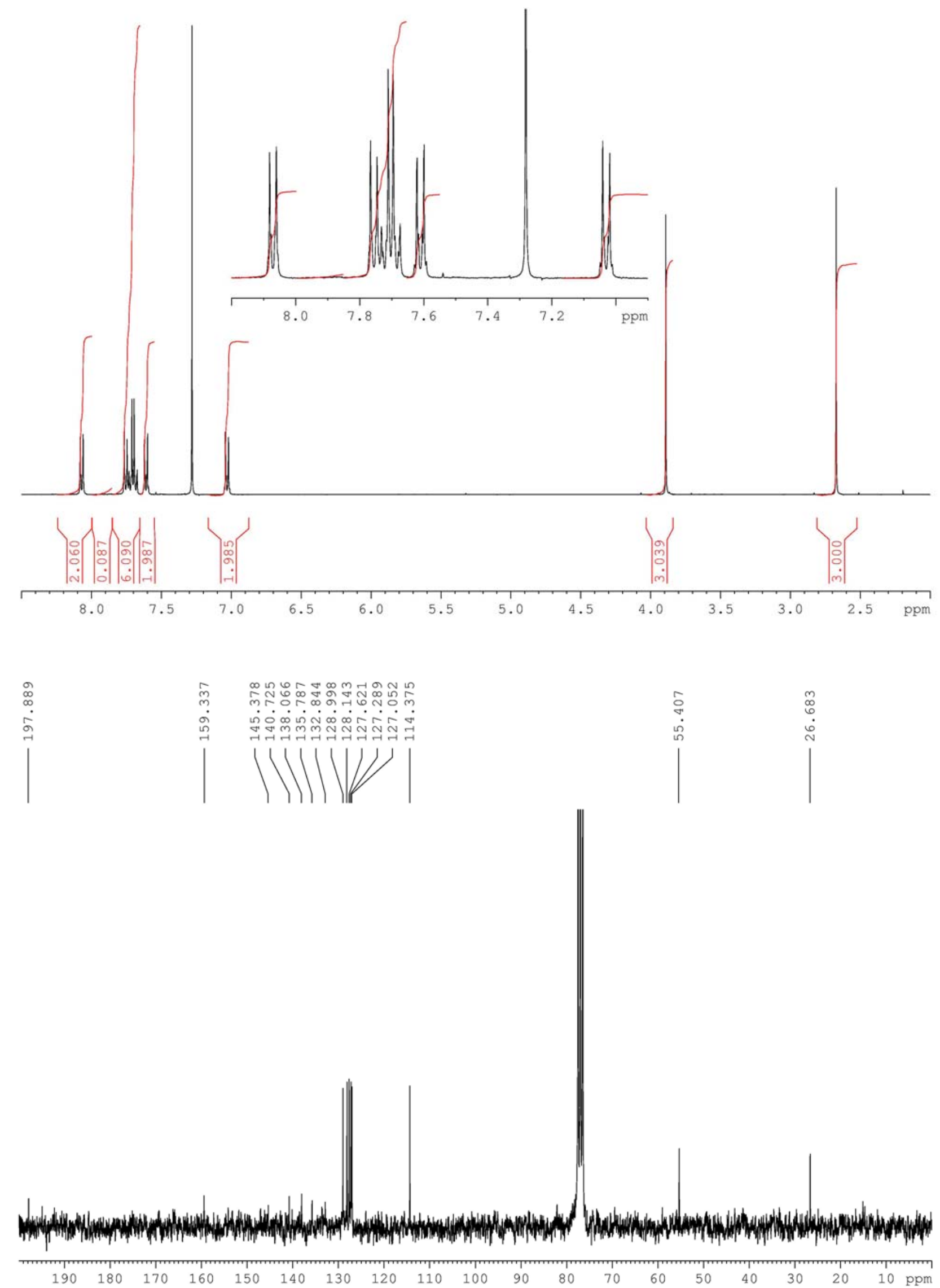
Figure S6. ${ }^{1} \mathrm{H}$ and ${ }^{13} \mathrm{C}$ NMR spectra of compound $3 g$.
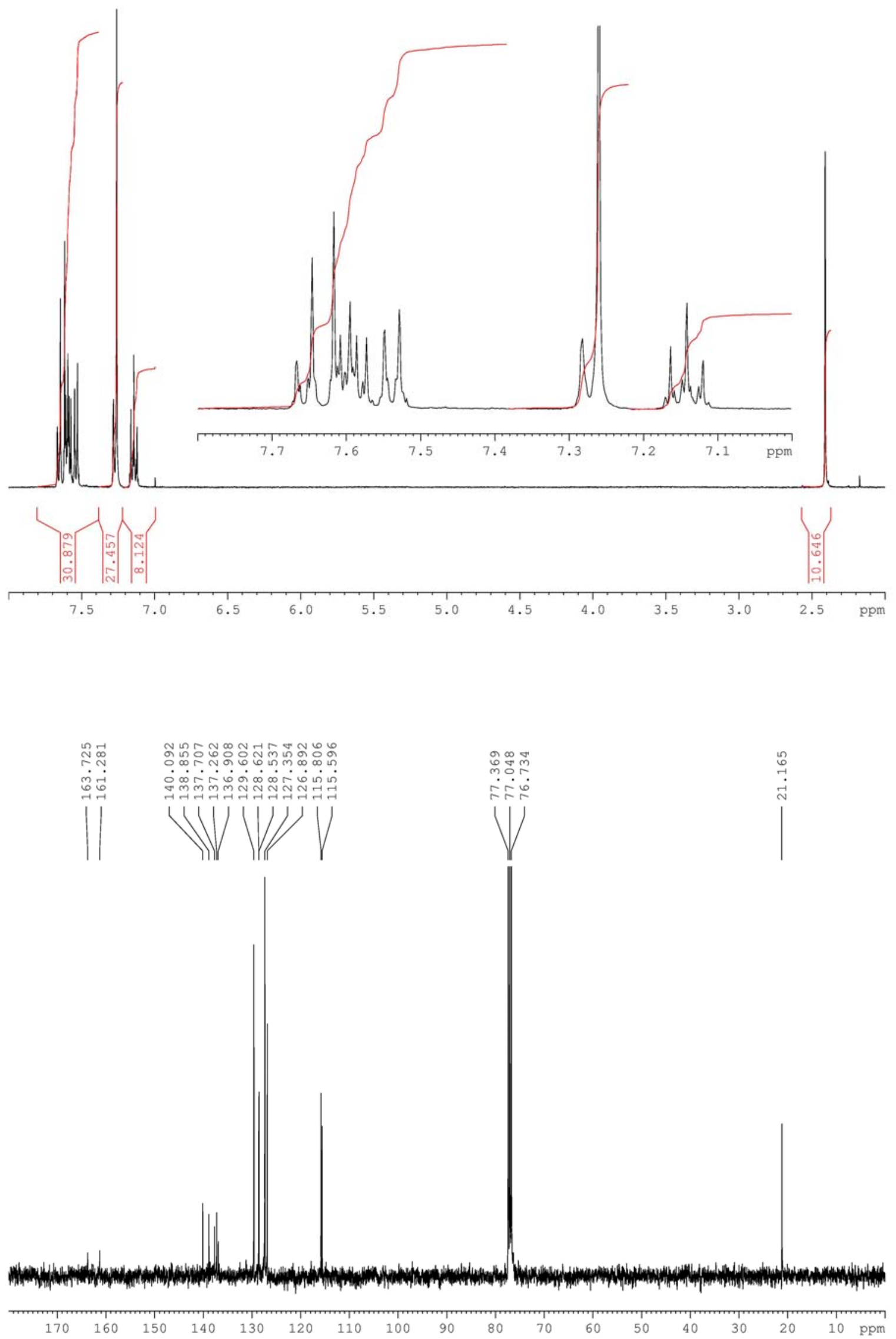
Figure S7. ${ }^{1} \mathrm{H}$ and ${ }^{13} \mathrm{C}$ NMR spectra of compound $3 \mathbf{h}$.
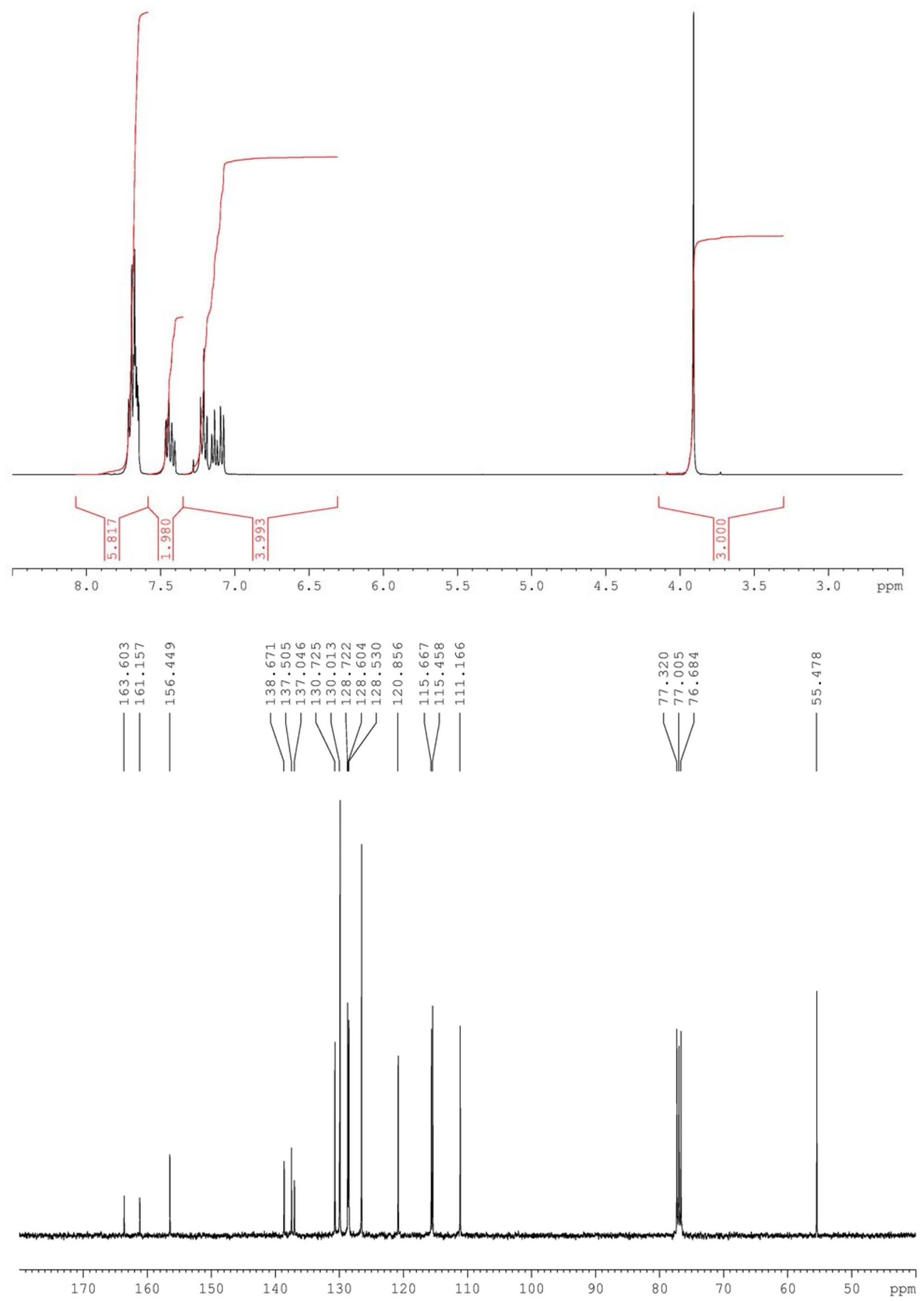
Figure S8. ${ }^{1} \mathrm{H}$ and ${ }^{13} \mathrm{C}$ NMR spectra of compound $\mathbf{3 k}$.
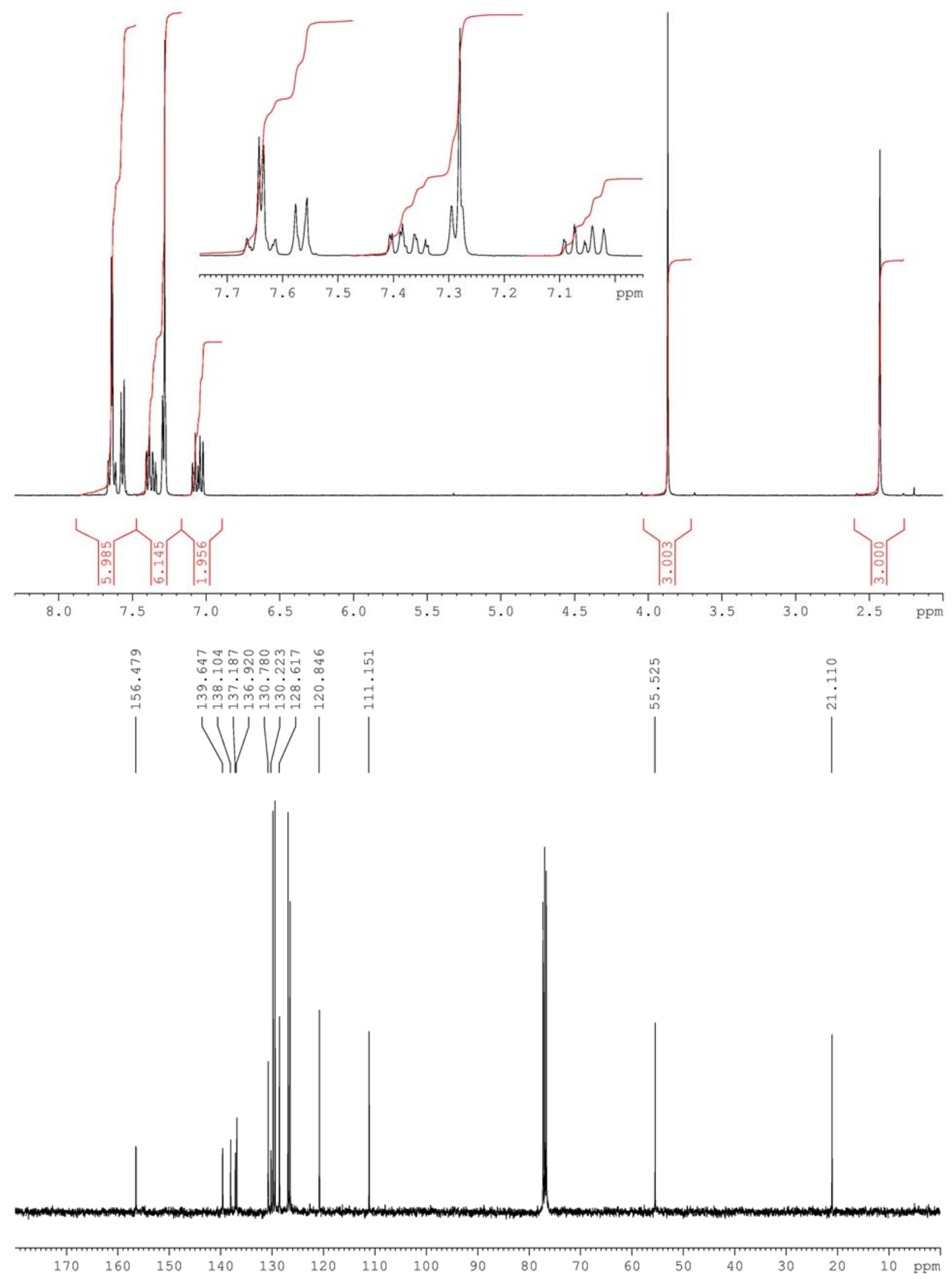
Figure S9. ${ }^{1} \mathrm{H}$ and ${ }^{13} \mathrm{C}$ NMR spectra of compound $\mathbf{4 a}$.
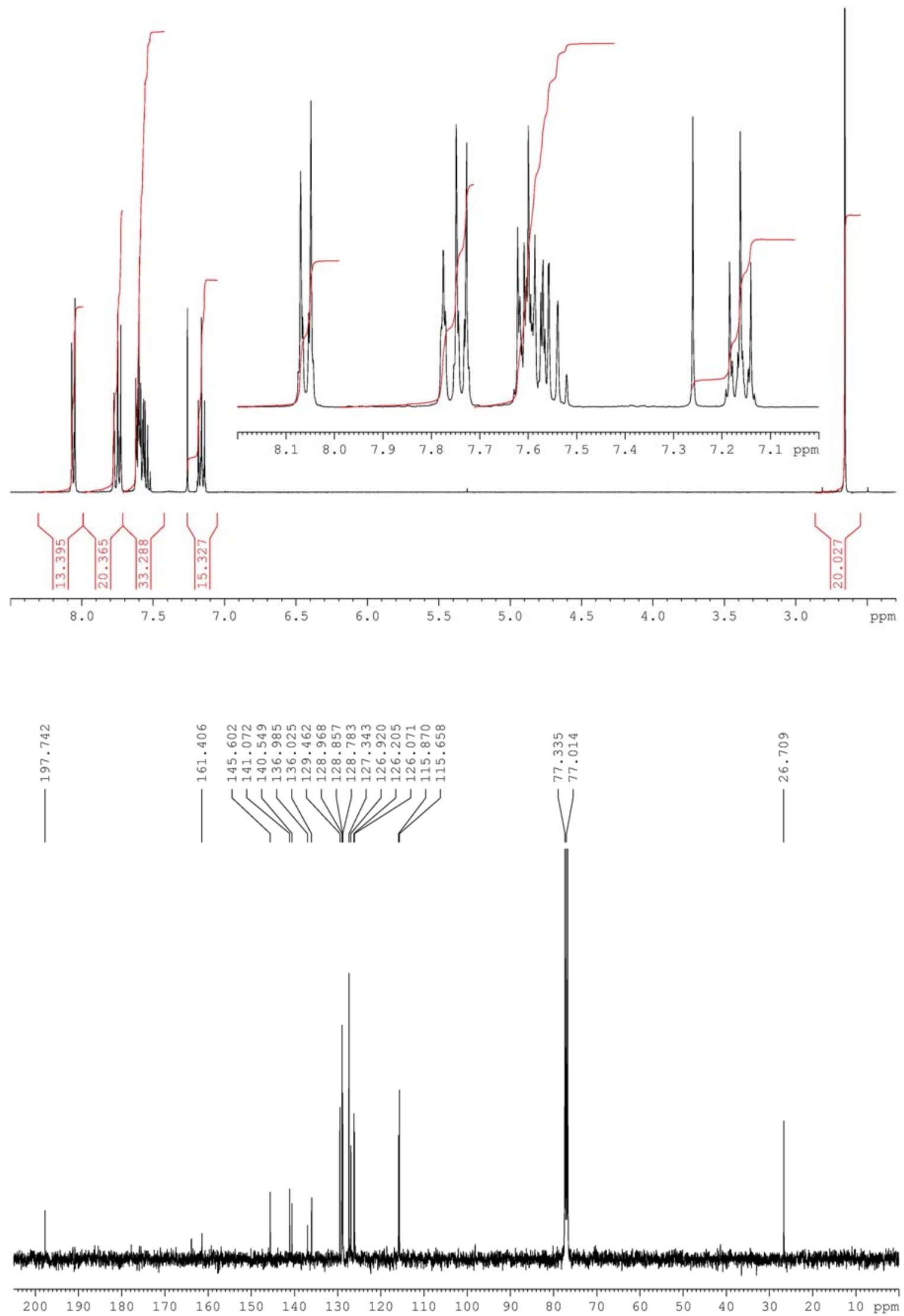
Figure S10. ${ }^{1} \mathrm{H}$ and ${ }^{13} \mathrm{C}$ NMR spectra of compound 4c.
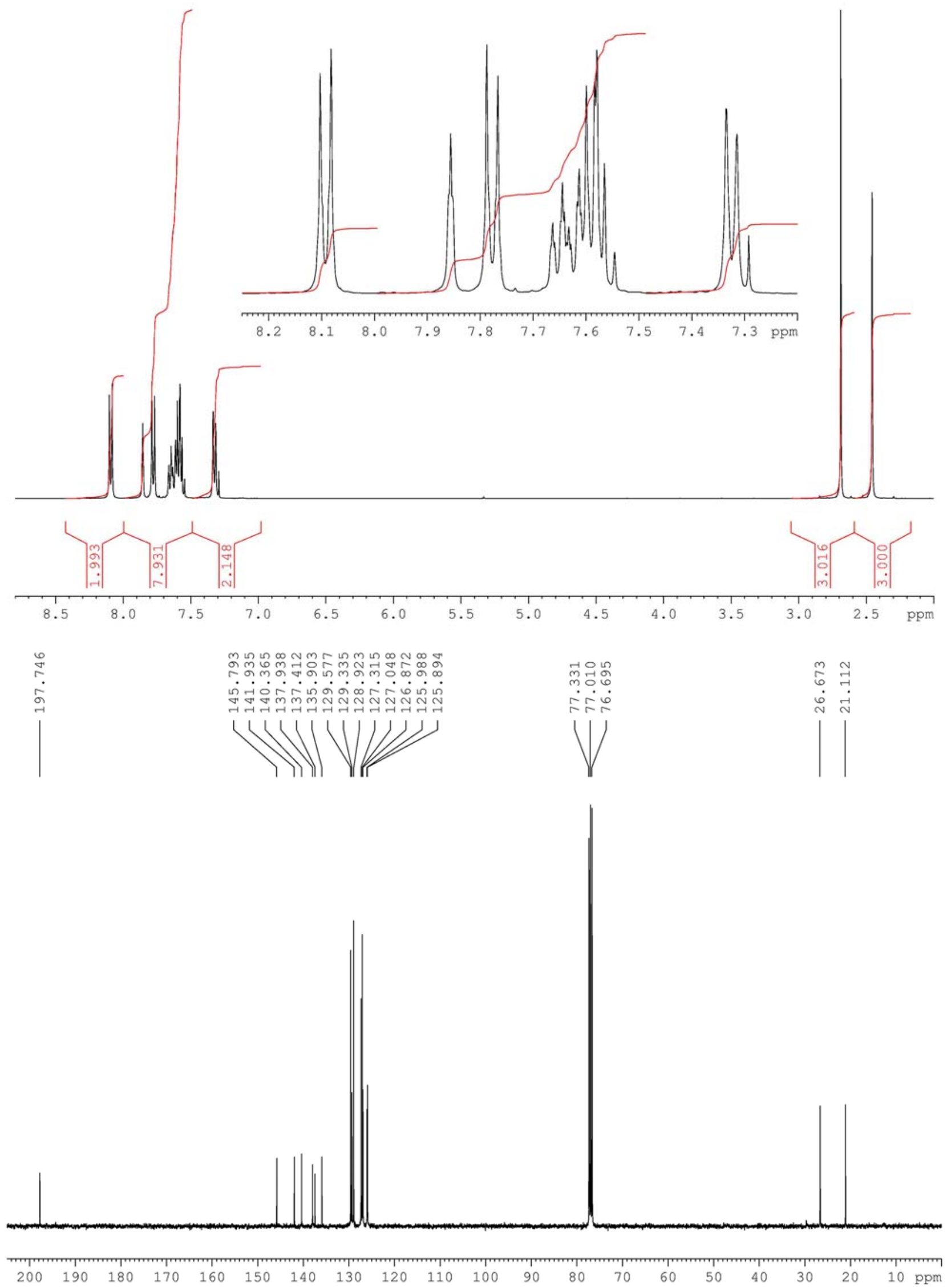
Figure S11. ${ }^{1} \mathrm{H}$ and ${ }^{13} \mathrm{C}$ NMR spectra of compound $\mathbf{4 d}$.
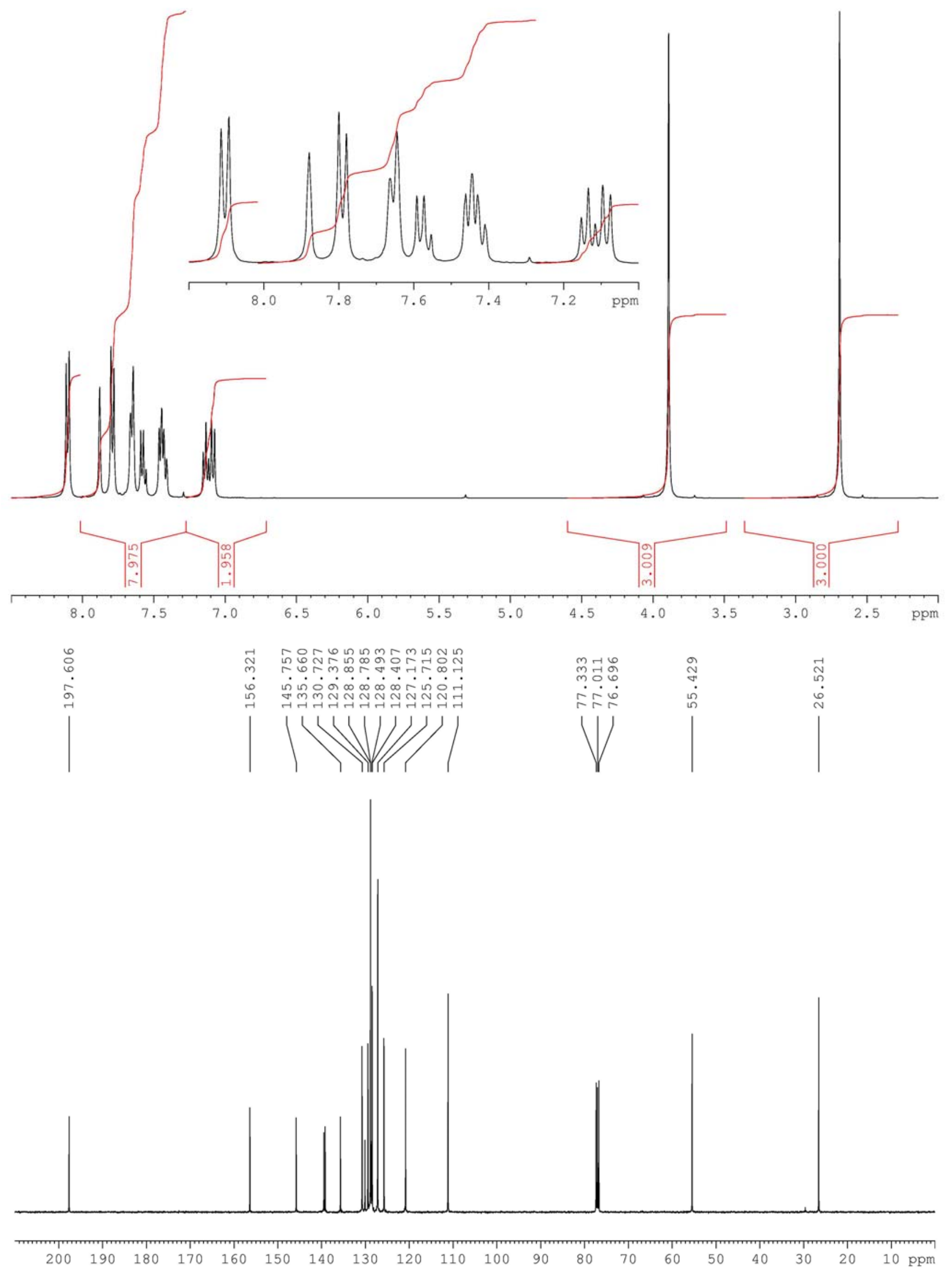
Figure S12. ${ }^{1} \mathrm{H}$ and ${ }^{13} \mathrm{C}$ NMR spectra of compound $4 \mathbf{e}$.
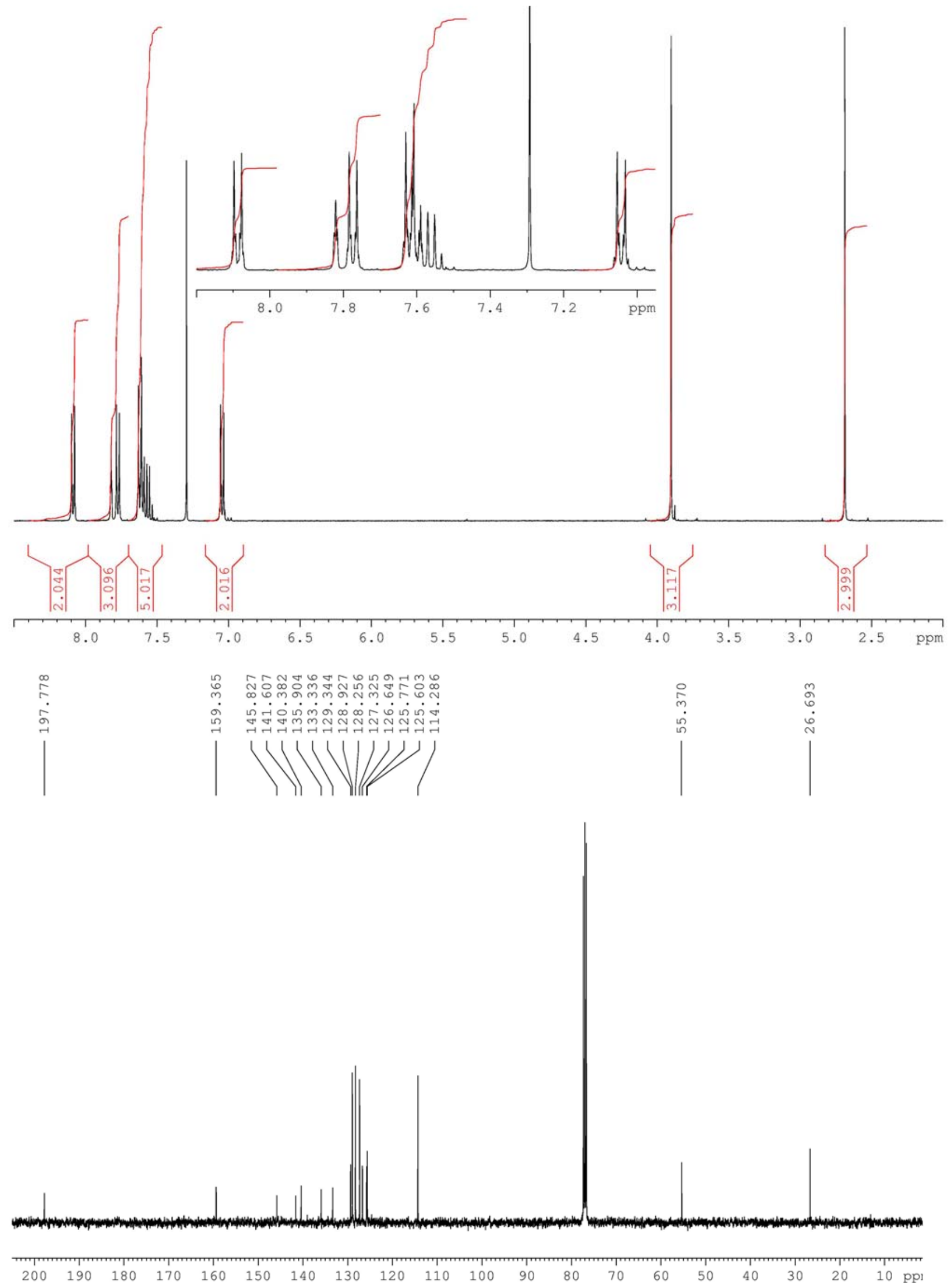
Figure S13. ${ }^{1} \mathrm{H}$ and ${ }^{13} \mathrm{C}$ NMR spectra of compound $\mathbf{4 f}$.
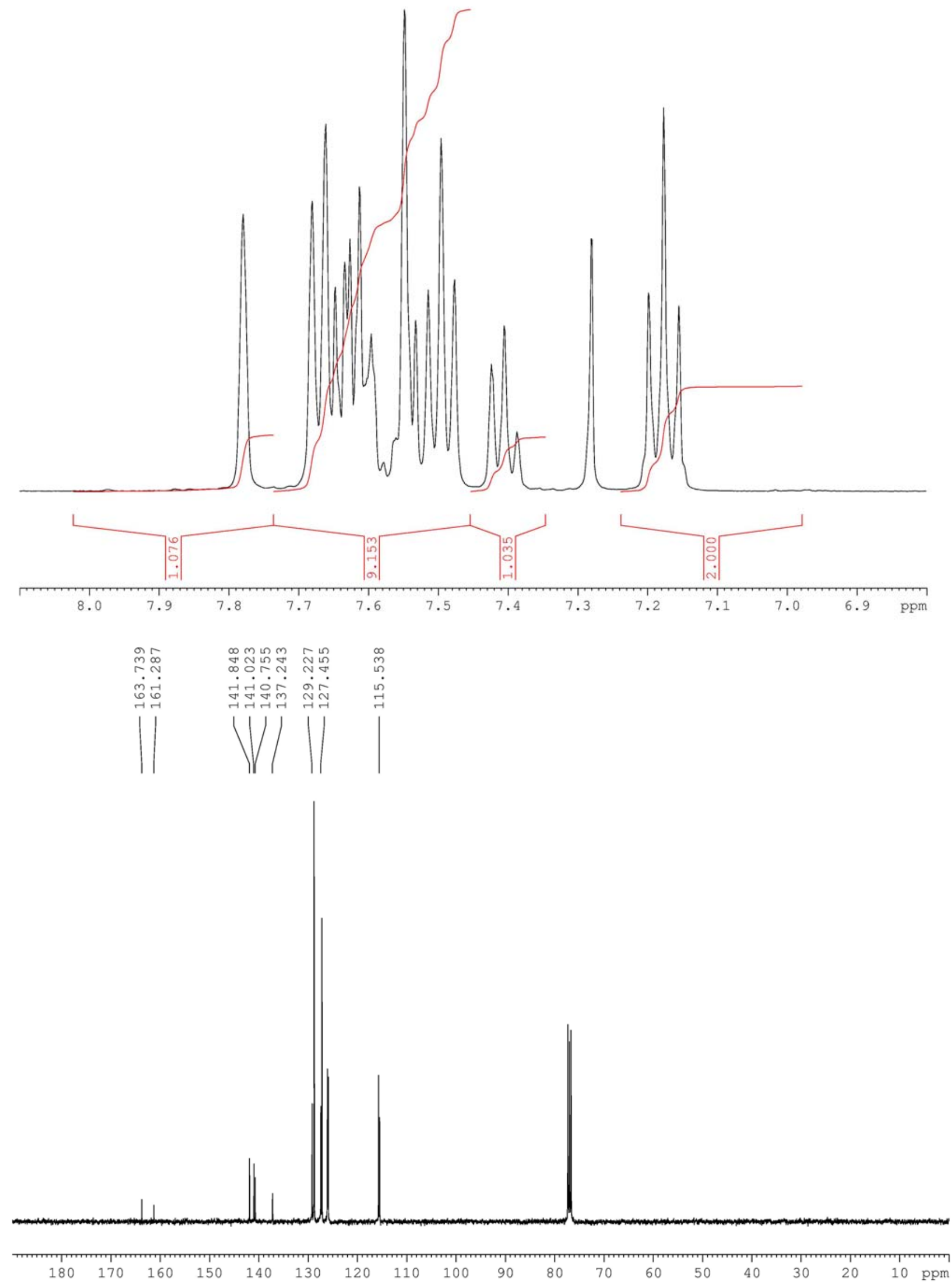
Figure S14. ${ }^{1} \mathrm{H}$ and ${ }^{13} \mathrm{C}$ NMR spectra of compound $\mathbf{4 g}$.
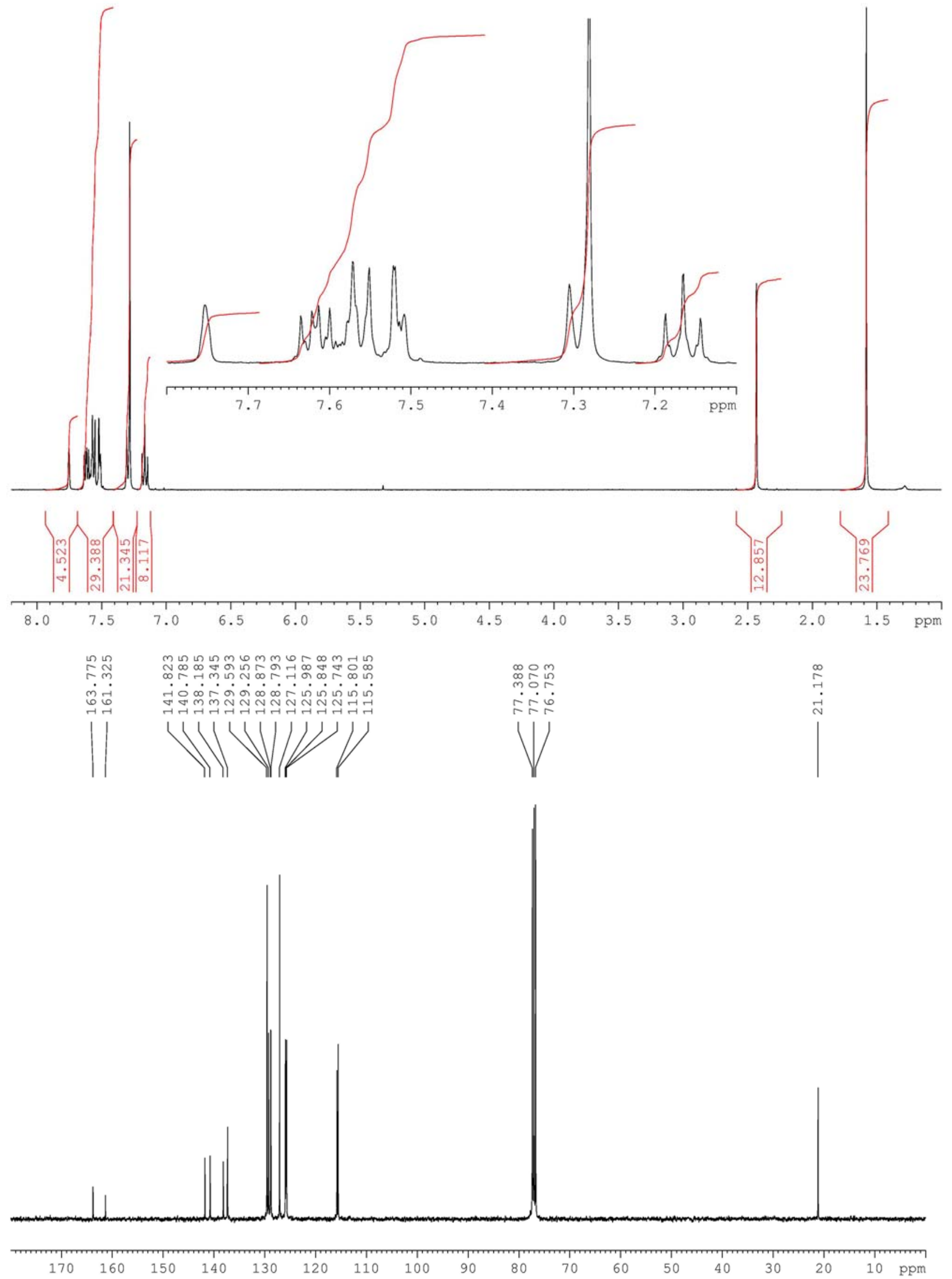
Figure S15. ${ }^{1} \mathrm{H}$ and ${ }^{13} \mathrm{C}$ NMR spectra of compound $\mathbf{4 h}$.
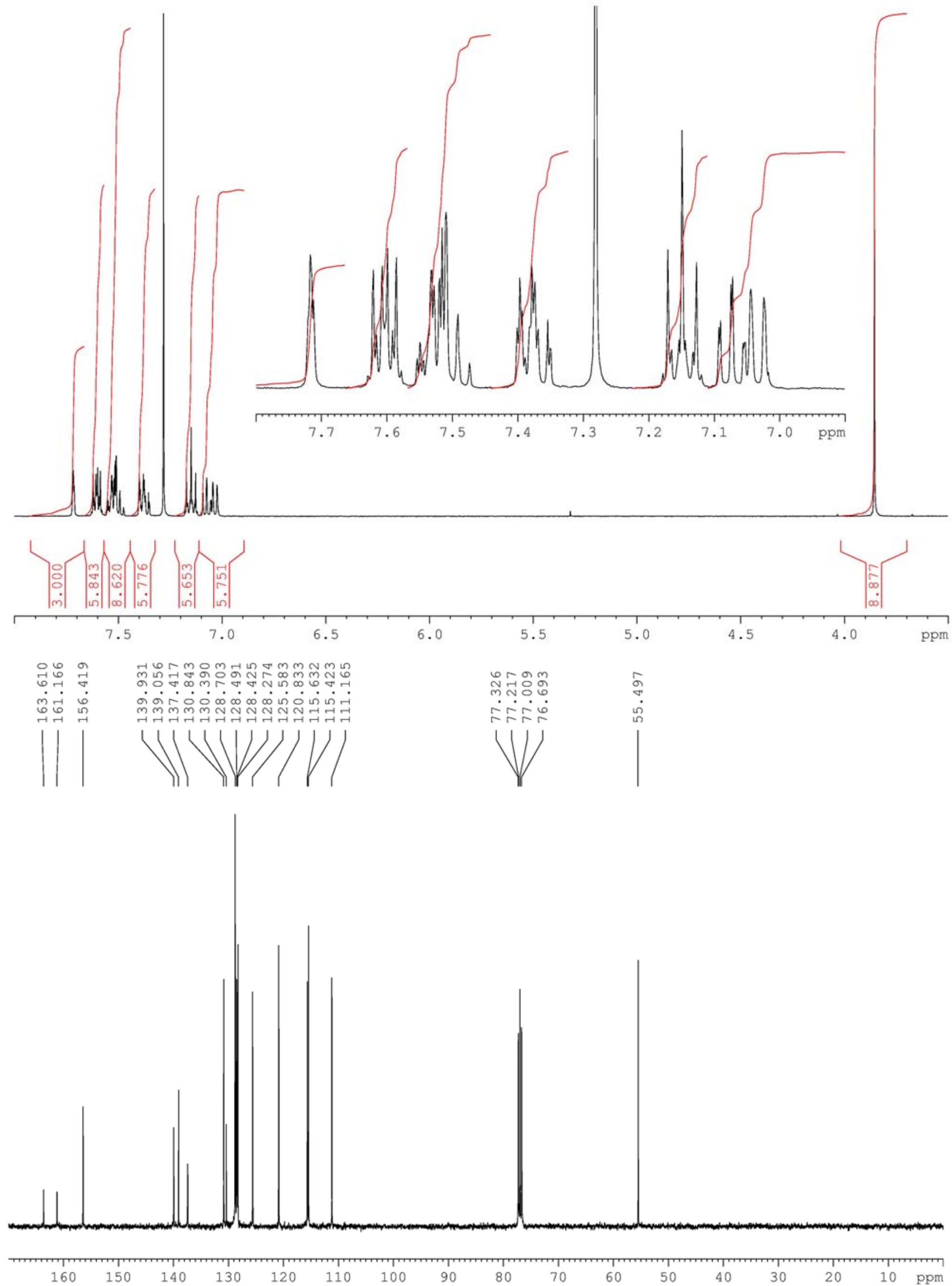
Figure S16. ${ }^{1} \mathrm{H}$ and ${ }^{13} \mathrm{C}$ NMR spectra of compound $\mathbf{4 j}$.
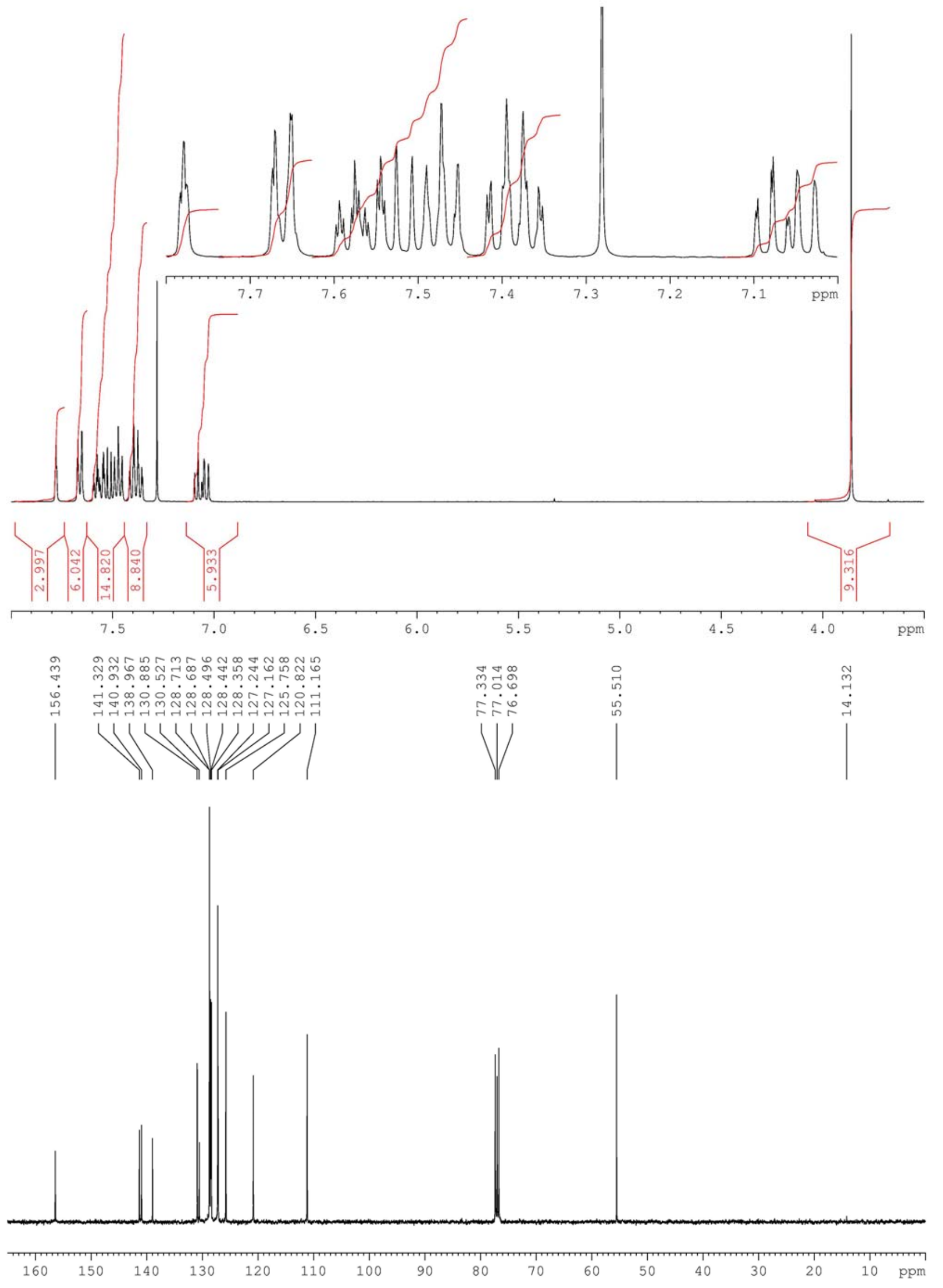
Figure S17. ${ }^{1} \mathrm{H}$ and ${ }^{13} \mathrm{C}$ NMR spectra of compound $\mathbf{4 k}$.
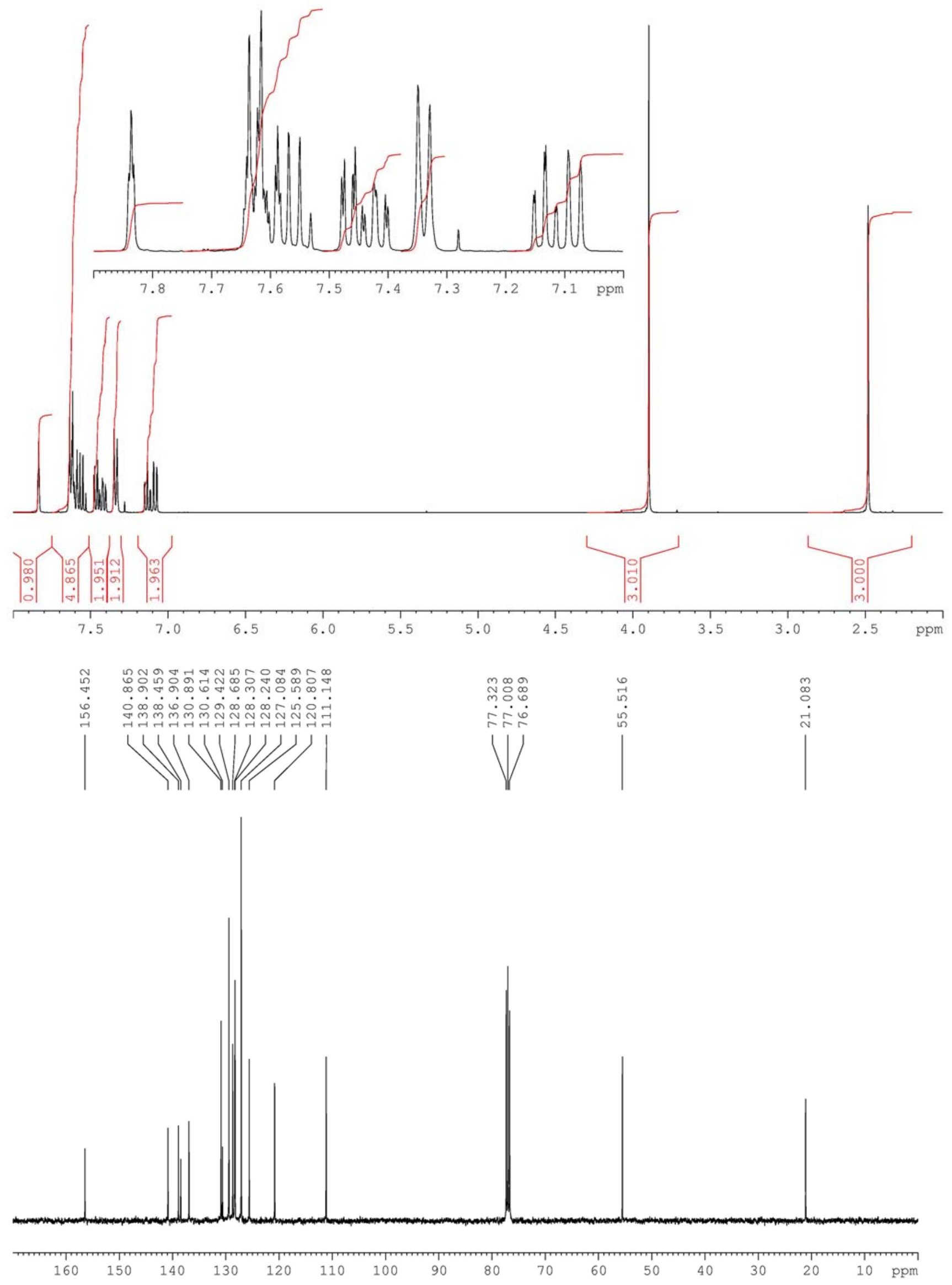
Figure S18. ${ }^{1} \mathrm{H}$ and ${ }^{13} \mathrm{C}$ NMR spectra of compound $5 \mathbf{e}$.
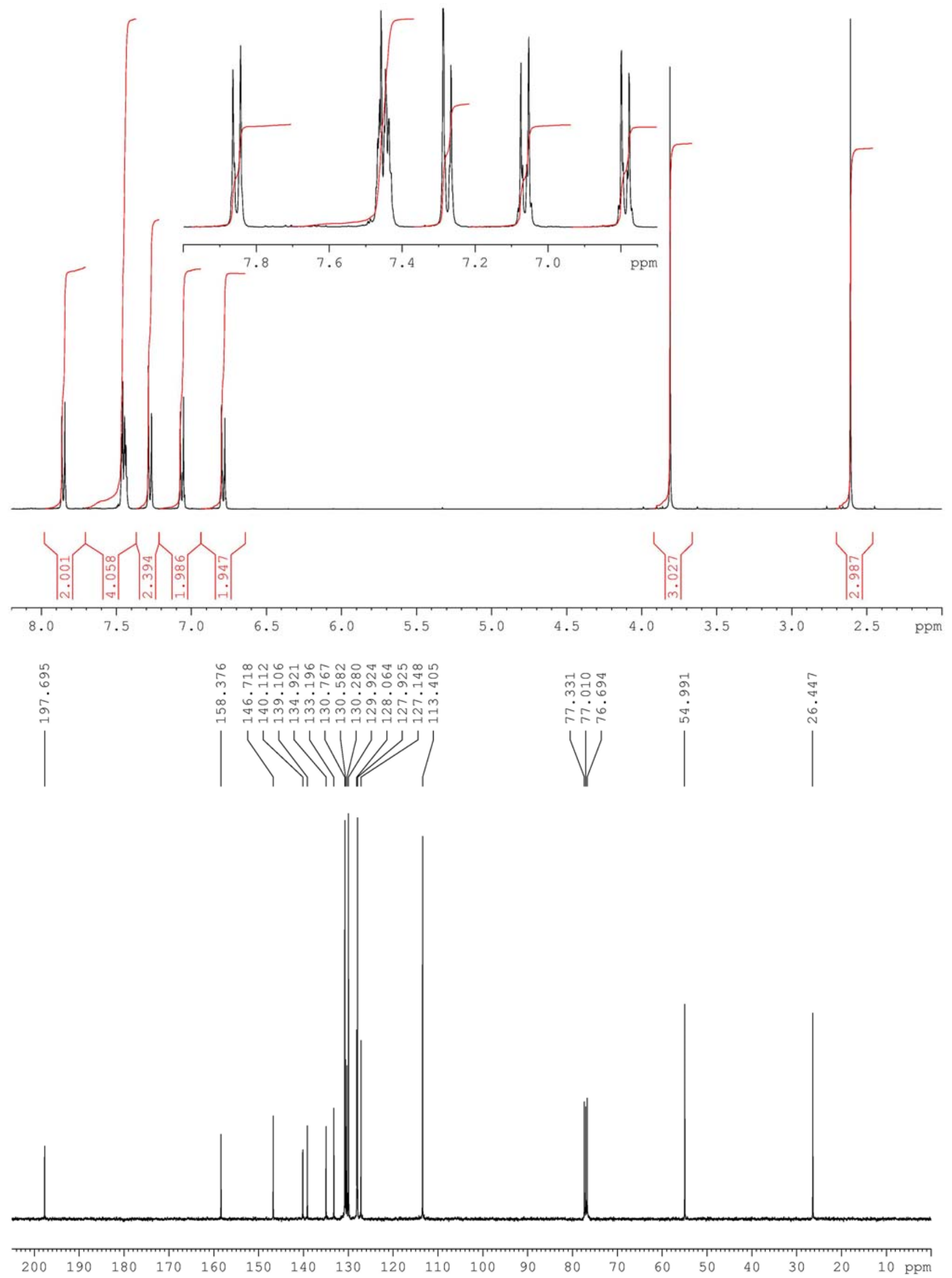
Figure S19. ${ }^{1} \mathrm{H}$ and ${ }^{13} \mathrm{C}$ NMR spectra of compound $\mathbf{5 f}$.
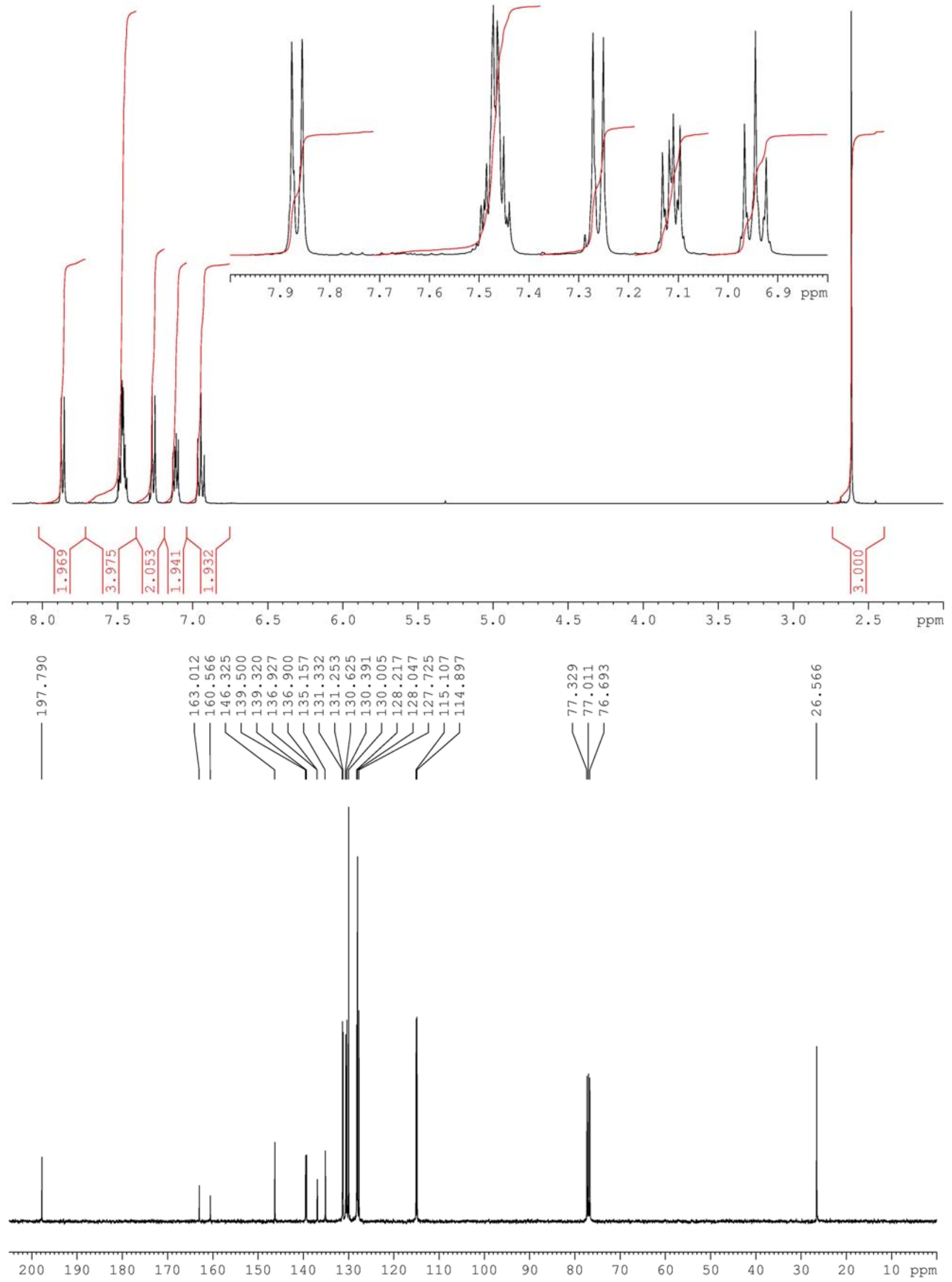
Figure S20. ${ }^{1} \mathrm{H}$ and ${ }^{13} \mathrm{C}$ NMR spectra of compound $\mathbf{5 g}$.
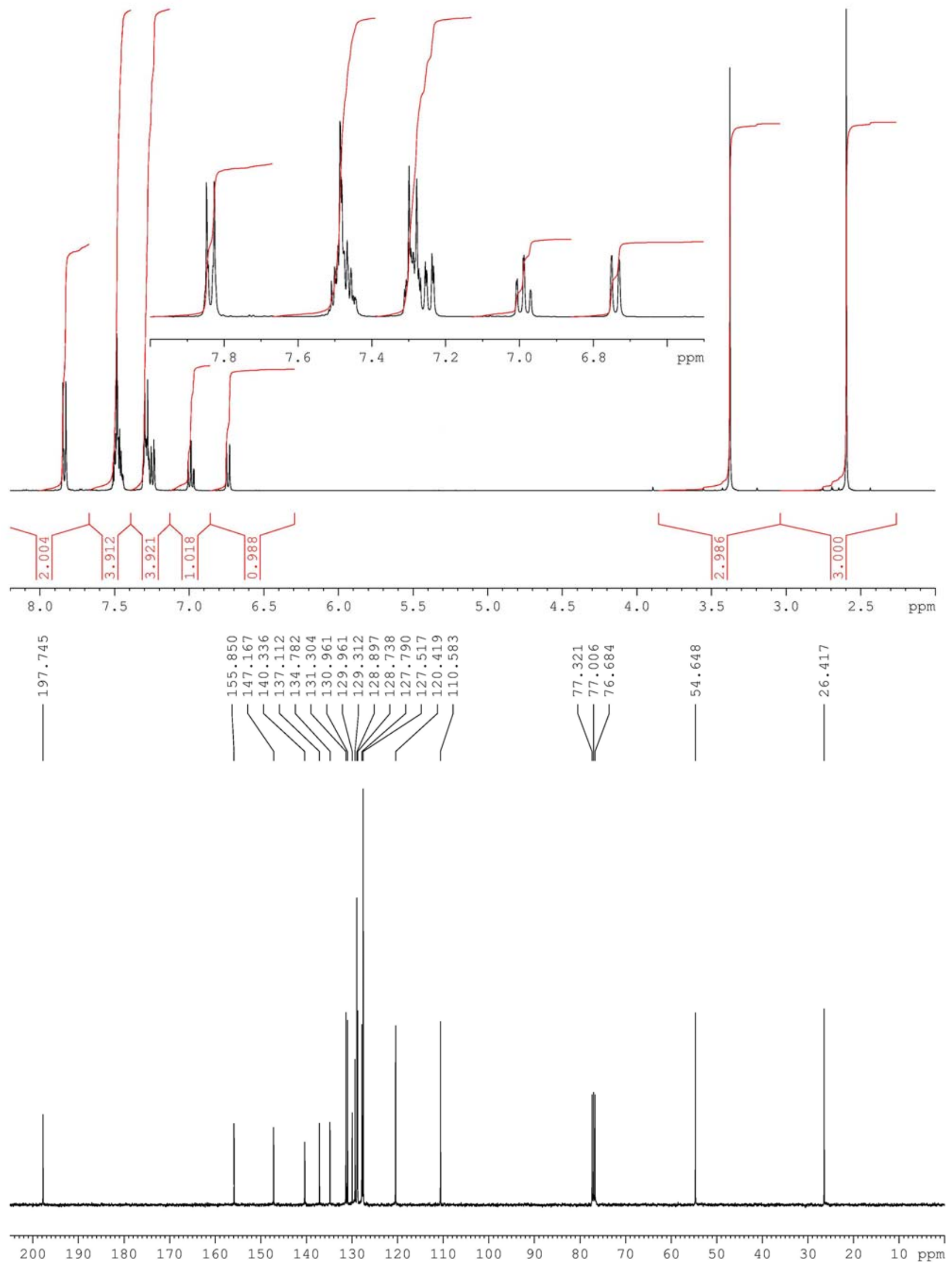
Figure S21. ${ }^{1} \mathrm{H}$ and ${ }^{13} \mathrm{C}$ NMR spectra of compound $5 \mathbf{h}$.
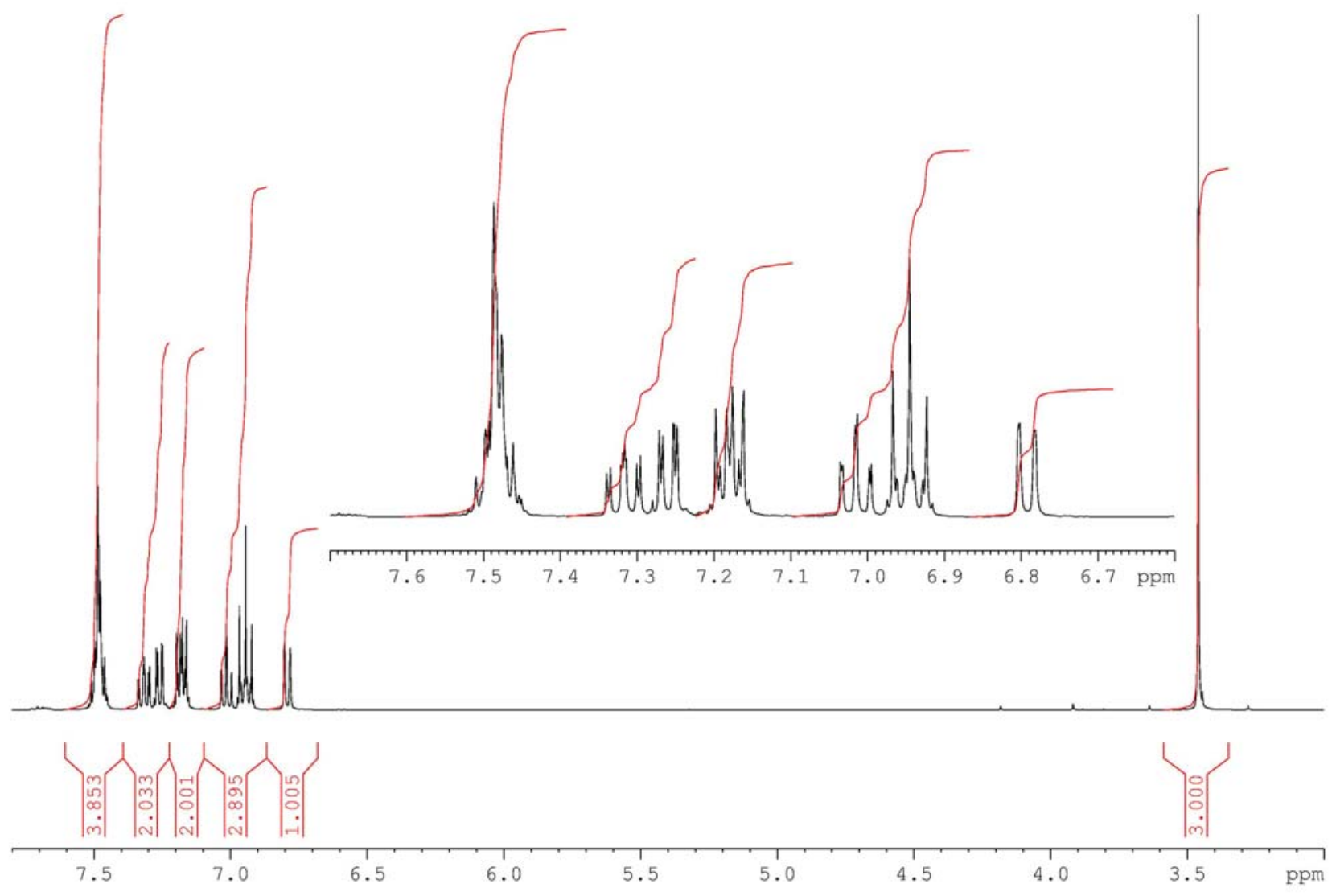

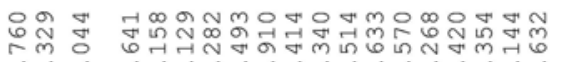

نิ0

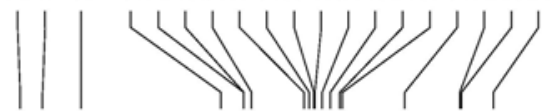

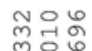

$\therefore \dot{0}$

VI

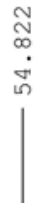
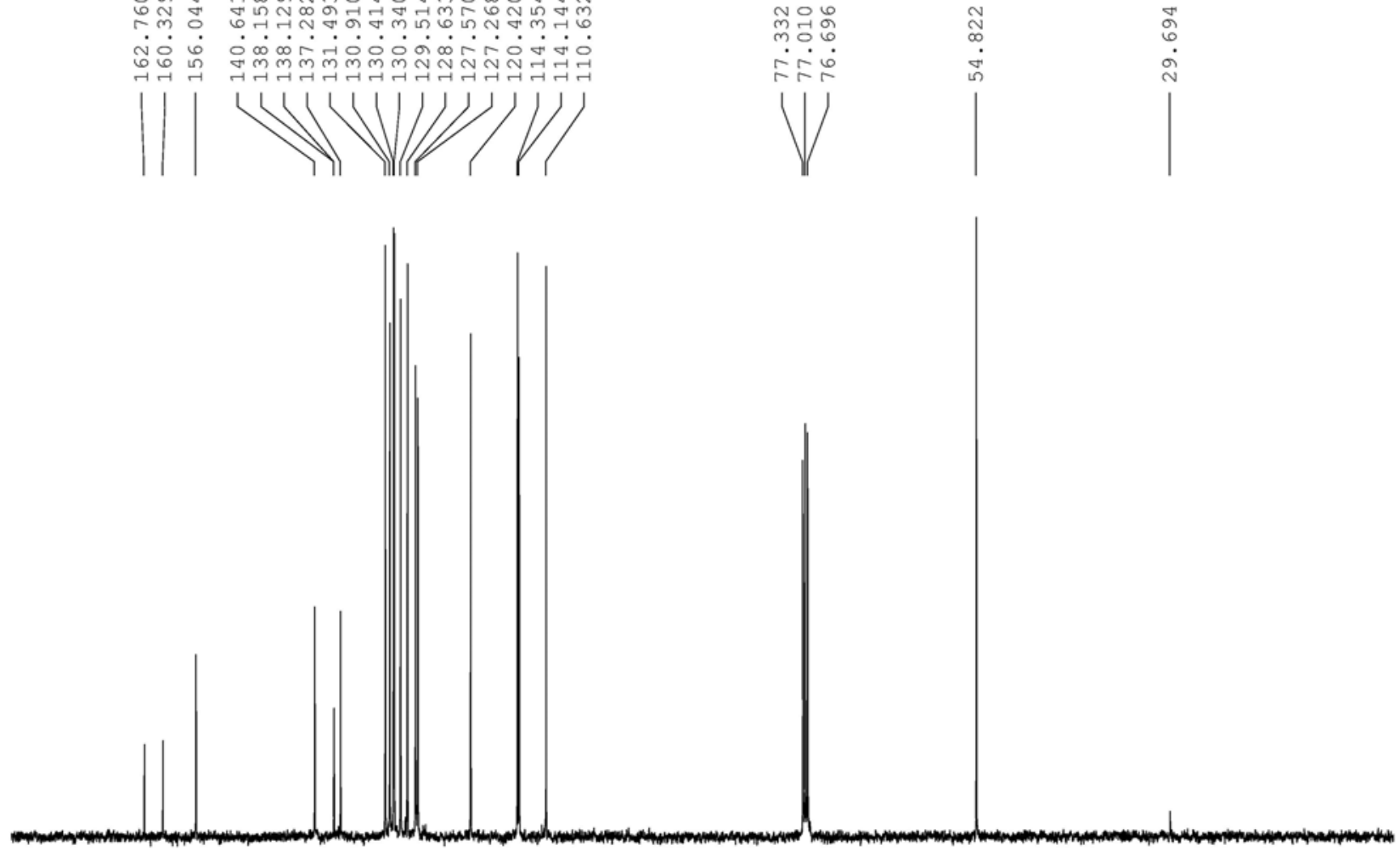

$170 \quad 160$

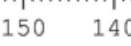

$130 \quad 120$

110

100
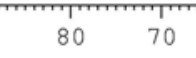

50

40

30 
Figure S22. ${ }^{1} \mathrm{H}$ and ${ }^{13} \mathrm{C}$ NMR spectra of compound $\mathbf{5 i}$.
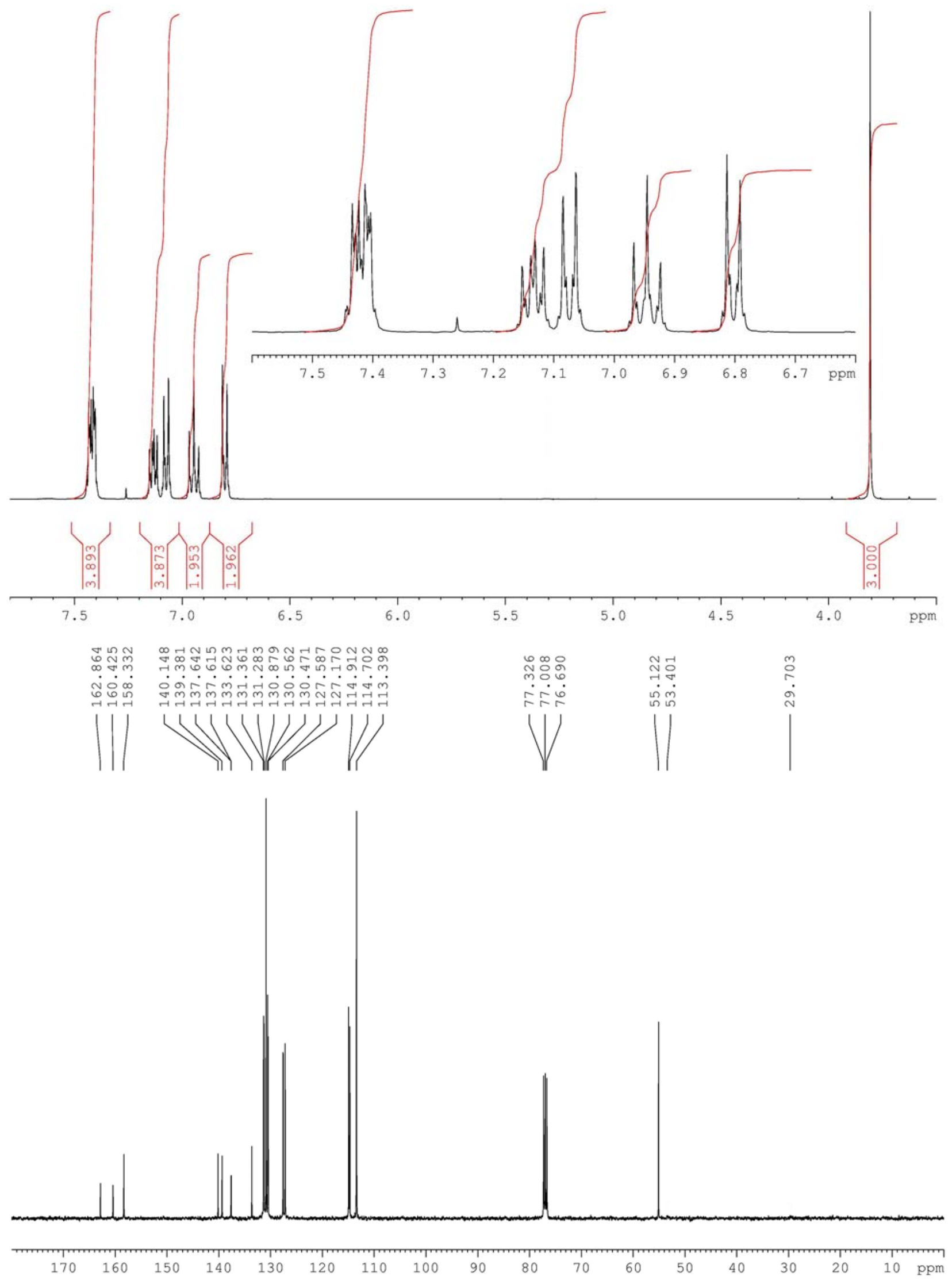
Figure S23. ${ }^{1} \mathrm{H}$ and ${ }^{13} \mathrm{C}$ NMR spectra of compound $\mathbf{5 j}$.
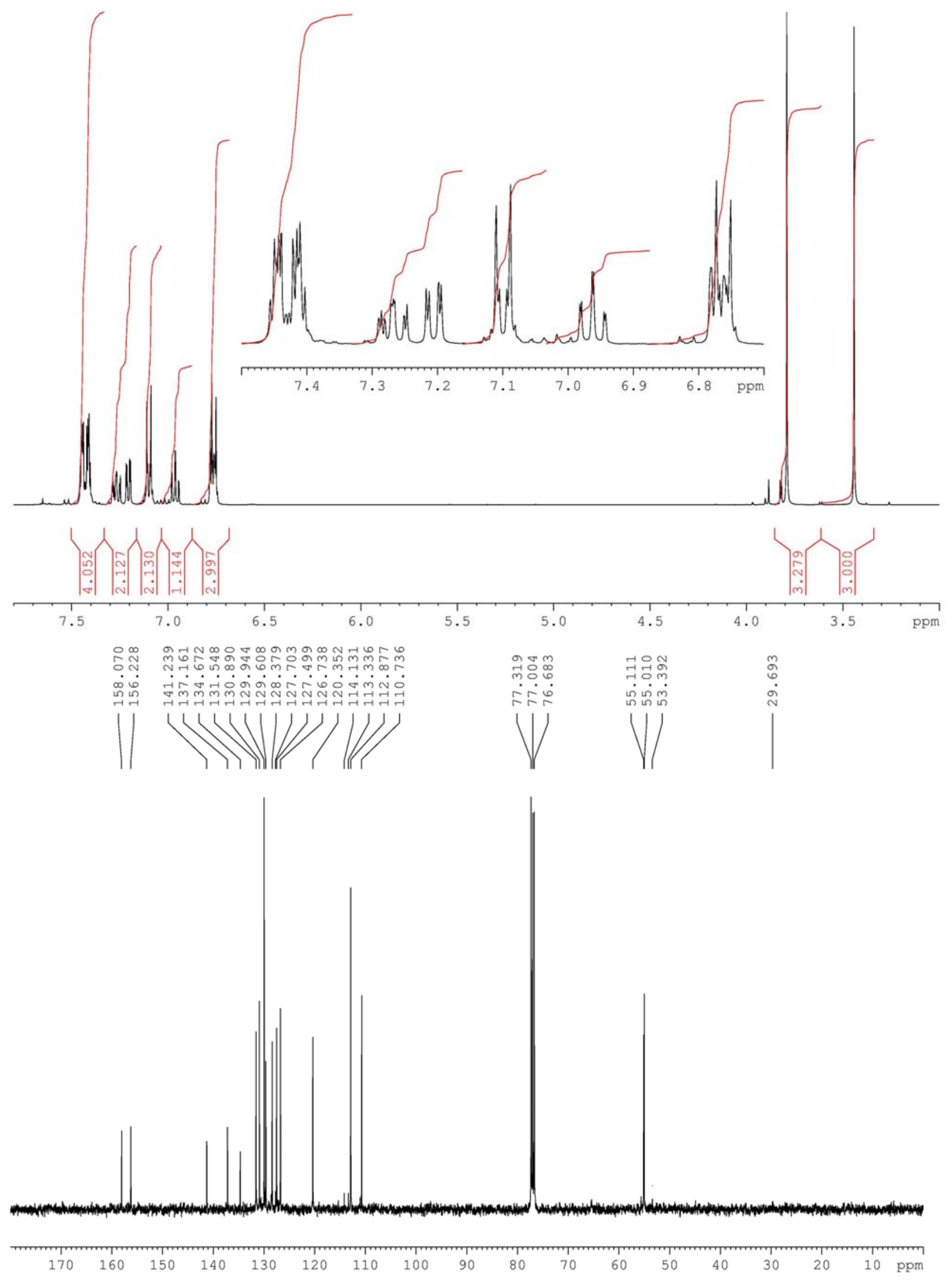


\section{References}

1. Byron, D. J.; Gray, G. W.; Wilson, R. C. J. Chem. Soc. C. 1966, 840.

2. Steiniger, B.; Wuest, F. R. J. Labelled Compd. Radiopharm. 2006, 49, 817.

3. De Luca, G.; Renzi, G.; Cipollini, R.; Pizzabiocca, A. J. Chem. Soc. Perkin Trans. 1 1980, 1901.

4. Gomberg, M. ; Pernert, J. C. J. Am. Chem. Soc. 1926, 48, 1381.

5. Bayer, U. ; Brune, H. A. Z. Naturforsch. B. Anorg. Chem. Org. Chem. 1983, 38, 226.

6. Sahoo, A. K. ; Oda, T.; Nakao, Y.; Hiyama, T. Adv. Synth. Catal. 2004, 346, 1715.

7. Ito, R. ; Migita, T. ; Morikawa, N.; Simamura, O. Bull. Chem. Soc. Jpn. 1963, 36, 992.

8. De Luca, G.; Renzi, G.; Cipollini, R.; Pizzabiocca, A. J. Chem. Soc. Perkin Trans. 1 1980, 1901.

9. Beadle, J. R.; S. H. Korzeniowsky, D. E. Rosenberg, B. J. Garcia-Slanga, G. W. Gokel, J. Org. Chem. 1984, 49, 1594.

10. Ito, R.; Migita, T.; Morikawa, N.; Okuni, M.; Simamura, O. Bull. Chem. Soc. Jpn. 1963, 36, 985.

11. Marchalin, S.; Kuthan, J. Collect. Czech. Chem. Commun. 1985, 50, 1862.

12. Song, Q. B.; Lin, R. X.; Teng, M. Y.; Zhang, J.; Ma, C. A. Synthesis 2006, 123.

13. Brocklehurst, P.; Buraway, A.; Thompson, A. R. Tetrahedron 1960, 10, 102.

14. Cho, C. H.; Sun, M.; Seo, Y. S.; Kim, C. B.; Park, K. J. Org. Chem. 2005, 70, 1482.

15. France, H.; Heilbron, Hey, D. H. J. Chem. Soc. 1939, 1288. 
${ }^{16}$. Sato, T.; Shimada, S.; Hata, K. Bull Chem. Soc. Jpn. 1969, 42, 766.

17. Hori, M.; Kataoka, T.; Shimizu, H.; Miyagaki, M. Chem. Pharm. Bull. 1974, 22, 2020.

18. Allen, C. F. H.; Burness, D. M. J. Org. Chem. 1949, 14, 163.

19. Barder, T. E.; Walker, S. D.; Martinelli, J. R.; Buchwald, S. L. J. Am. Chem. Soc. 2005, 127, 4685. 